Geophysical and Astrophysical Fluid Dynamics

Vol. 00, No. 00, 00 Month 2012, 1-28

\title{
Frequency staircases in narrow-gap spherical Couette flow
}

\author{
ANDREW M. SOWARD $\dagger^{*}$ and ANDREW P. BASSOM $\ddagger$ \\ $\dagger$ School of Mathematics and Statistics, Newcastle University, Newcastle upon Tyne, NE1 7RU, UK \\ $\ddagger$ School of Physical Sciences, University of Tasmania, Private Bag 37, Hobart 7001, Tasmania, \\ Australia
}

(Received 19 April 2015; in revised form 13 October 2015; first published online ????)

\begin{abstract}
Recent studies of plane parallel flows have emphasised the importance of finite-amplitude self-sustaining processes for the existence of alternative non-trivial solutions. The idea behind these mechanisms is that the motion is composed of distinct structures that interact to self-sustain. These solutions are not unique and their totality form a skeleton about which the actual realised motion is attracted. Related features can be found in spherical Couette flow between two rotating spheres in the limit of narrow-gap width. At lowest order the onset of instability is manifested by Taylor vortices localised in the vicinity of the equator. By approximating the spheres by their tangent cylinders at the equator, a critical Taylor number based on the ensuing cylindrical Couette flow problem would appear to provide a lowest order approximation to the true critical Taylor number. At next order, the latitudinal modulation of their amplitude $a$ satisfies the complex Ginzburg-Landau equation (CGLe)

$$
\partial a / \partial t=(\lambda+\mathrm{i} x) a+\partial^{2} a / \partial x^{2}-|a|^{2} a,
$$

where $-x$ is latitude scaled on the modulation length scale, $t$ is time and $\lambda$ is proportional to the excess Taylor number. The amplitude $a$ governed by our CGLe is linearly stable for all $\lambda$ but possesses non-decaying nonlinear solutions at finite $\lambda$, directly analogous to plane Couette flow. Furthermore, whereas the important balance $\partial a / \partial t=\mathrm{i} x a$ suggests that the Taylor vortices ought to propagate as waves towards the equator with frequency proportional to latitude, the realised solutions are found to exist as pulses, each locked to a discrete frequency, of spatially modulated Taylor vortices. Collectively they form a pulse train. Thus the expected continuous spatial variation of the frequency is broken into steps (forming a staircase) on which motion is dominated by the local pulse. A wealth of solutions of our CGLe have been found and some may be stable. Nevertheless, when higher-order terms are reinstated, solutions are modulated on a yet longer length scale and must evolve. So, whereas there is an underlying pulse structure in the small but finite gap limit, motion is likely to be always weakly chaotic. Our CGLe and its solution provides a paradigm for many geophysical and astrophysical flows capturing in minimalistic form
\end{abstract} interaction of phase mixing ixa, diffusion $\partial^{2} a / \partial x^{2}$ and nonlinearity $|a|^{2} a$.

Keywords: Couette flow, Taylor vortices, Narrow-gap limit, Frequency staircase for pulse packets, Selfsustaining processes

\section{Introduction}

The study of the onset of stability, as well as its transition to turbulence, in Couette flow or one of its variants has a long and celebrated history (see Manneville 2014, for recent perspectives). Cylindrical Couette flow has received considerable attention particularly because the configuration is amenable to accurate experimental study (Koschmieder 1993). As the appropriate Taylor number Ta is increased, so the laminar flow becomes unstable at some critical value $\mathrm{Ta}=\mathrm{Ta}_{\mathrm{cCf}}$ to the onset of axisymmetric Taylor vortices. The subsequent bifurcation to wavy vortices and other yet more complicated flows is well documented and understood. Though apparently simpler, plane Couette flow characterised by a Reynolds number Re possesses the intriguing complication that the flow lacks any critical value $\operatorname{Re}_{c}$, i.e., the flow is stable to

*Corresponding author. Email: andrew.soward@ncl.ac.uk 
all infinitesimal perturbations for all Re. The determination of finite amplitude solutions at finite Re has provided a major challenge that in recent years has been addressed successfully (Nagata 1990, Clever and Busse 1992, 1997, Waleffe 1997, 2003) using homotopy methods.

A variant of the cylindrical Couette flow configuration that has received relatively little attention is that of spherical Couette flow (see, e.g., Marcus and Tuckerman 1987). In this problem the space between concentric spherical shells is filled with viscous fluid and the shells rotated about a common axis thorough their centres. Should the gap between the inner and outer spheres be small, the Taylor vortices that occur at the onset of instability are located near the equator, i.e., where the centrifugal acceleration is maximum. Experimentally this has the attractive feature that the vortices have negligible amplitude near the poles, i.e., where the centrifugal acceleration vanishes. This means the flow is not bothered by end effects as it is in cylindrical Couette flow. Unfortunately in the narrow-gap case for which the ratio $\varepsilon$ of gap width to shell radius is small, a difficult asymptotic problem emerges involving three distinct latitudinal length scales (Bassom and Soward 2004). In units of the shell radius, axisymmetric Taylor vortices on the shortest length scale $\ell_{c}$, roughly the shell gap width, are confined near the equator. They are locally unstable over a relatively broad latitudinal extent $\theta_{\text {us }}$, where their amplitude is modulated and governed by a full Complex Ginzburg Landau equation (CGLe) which also involves the small parameter $\varepsilon$. Though $\theta_{u s}$ is the longest length scale, it is still small compared to the shell radius. The lowest-order approximation to the full CGLe on an intermediate phase mixing length scale yields the simpler (and fundamental to the entire solution) equation

$$
\frac{\partial a}{\partial t}=(\lambda+\mathrm{i} x) a+\frac{\partial^{2} a}{\partial x^{2}}-|a|^{2} a,
$$

for the complex amplitude $a$. Here $-x$ is the scaled latitude while the constant $\lambda$ measures, in appropriate scaled units, the excess $\mathrm{Ta}-\mathrm{Ta}_{c}$ above the speculated critical value

$$
\mathrm{Ta}_{c} \equiv \mathrm{Ta}_{\mathrm{cCf}},
$$

that is the critical Taylor number appropriate to the cylindrical Couette flow problem. It is unsurprising that the equatorial region is well approximated by two rotating cylinders when $\varepsilon$ is small, and for some time it was assumed that the critical Taylor number for the finite-gap spherical Couette flow problem, $\mathrm{Ta}_{\mathrm{sCf}}$, should approach $\mathrm{Ta}_{\mathrm{cCf}}$ as $\varepsilon \downarrow 0$. It was not until the analysis of Soward and Jones (1983) that it was realised that $\mathrm{Ta}_{\mathrm{SCf}}-\mathrm{Ta}_{\mathrm{cCf}}>0$, even as $\varepsilon \downarrow 0$. It is this aspect that makes the use of the phrase 'appropriate scaled units' vital to our understanding of the bounded solutions of (1). The value of $\lambda\left(\propto \mathrm{Ta}_{\mathrm{sCf}}-\mathrm{Ta}_{\mathrm{cCf}}\right)$ tends to infinity in concert with $\varepsilon$ decreasing to zero so that, in particular, equation (1) does not even apply at $\mathrm{Ta}_{\mathrm{sCf}}$ !

Three features of (1) stemming from the term ixa ought to be made clear at this early stage.

(i) Though the explicit dependence on $x$ implies that solutions are not translationary invariant they have the useful related property that, if $a(x, t)$ is a solution, then so is

$$
\mathrm{e}^{\mathrm{i} \Omega t} a(x-\Omega, t),
$$

for any constant frequency $\Omega$.

(ii) A curious feature of (1) is that the leading-order balance for large $|x|$ is

$$
\frac{\partial a}{\partial t}=\mathrm{i} x a \quad \Longrightarrow \quad a \propto \exp (\mathrm{i} x t) .
$$

This type of solution, for which the frequency varies continuously with position $x$ is often referred to as "phase mixing".

(iii) The CGLe (1) possesses no critical value $\lambda_{c}$ at which the trivial solution $a=0$ bifurcates to a finite amplitude solution $a \neq 0$ bounded as $|x| \rightarrow \infty$. 
The aim of this paper is largely to discuss the relevance of (1) to the narrow-gap spherical Couette flow problem and it will be seen that many of the issues to be addressed have features in common with the fundamental problem of plane Couette flow. Indeed our underlying thesis is that the key ingredient that contributes to the lack of a critical Reynolds number in the plane Couette case is phase mixing, just as (1) possesses no $\lambda_{c}$ as noted in (iii) above. It is the absence of a critical $\lambda$, which motivates our use of the subscript ${ }_{c}$ in section 3 to denote values taken at $\mathrm{Ta}=\mathrm{Ta}_{\mathrm{cCf}}$, the critical Taylor number for cylindrical Couette flow (see (2)).

Though much of this paper is review by nature, but with some new results, we shall also highlight the connections and the relevance of our narrow-gap spherical Couette study, that builds on (1), to wide-ranging investigations that include the atmosphere (e.g., Farrell and Ioannou 1993), solar physics (e.g., De Moortel and Nakariakov 2012), planetary convection (see, e.g., Dormy et al. 2004, and references therein), MHD turbulence (e.g., Leprovost and Kim 2009), dynamos (e.g., Heinemann et al. 2011) and particularly accretion discs (see, e.g. Dubrulle and Knobloch 1992, Hawley et al. 1995, Riols et al. 2013) which exhibit Couette flow type motion. So despite the restricted application for which (1) was derived, this CGLe provides a paradigm for many geophysical and astrophysical flows capturing in minimalistic form the interaction of phase mixing, diffusion and nonlinearity.

\section{Background}

\subsection{Plane Couette flow}

Plane Couette flow (pCf) is best described relative to rectangular Cartesian coordinates $(x, y, z)$. The basic state consists of steady unidirectional motion $(U(y), 0,0)$ of a constant density fluid in the $x$-direction between plane boundaries located at $y= \pm L$ (say). When those boundaries move at speed $\pm S L$, the velocity is the linear shear $U(y)=S y$, with $S=\mathrm{d} U / \mathrm{d} y$ a constant. The associated Reynolds number is then

$$
\operatorname{Re}=S L^{2} / \nu,
$$

where $\nu$ is the kinematic viscosity. At time $\tau$ (to distinguish it from the scaled $t$ of section 1 ), small perturbations $\boldsymbol{u}^{\prime}, p^{\prime}$ to the velocity and pressure satisfy the linear equation

$$
\frac{\partial \boldsymbol{u}^{\prime}}{\partial \tau}+U \frac{\partial \boldsymbol{u}^{\prime}}{\partial x}=-\nabla p^{\prime}-S u_{y}^{\prime} \boldsymbol{e}_{x}+\nu \nabla^{2} \boldsymbol{u}^{\prime}, \quad \boldsymbol{\nabla} \cdot \boldsymbol{u}^{\prime}=0,
$$

where $\boldsymbol{e}_{x}$ is the unit vector in the $x$-direction, and are subject to the usual no-slip boundary conditions $\boldsymbol{u}^{\prime}=\mathbf{0}$ on $y= \pm L$.

For disturbances, spatially periodic in the $x-z$ plane, the horizontally averaged (denoted by an overbar) kinetic energy $\frac{1}{2} \overline{\boldsymbol{u}^{\prime 2}}$ satisfies

$$
\frac{\partial}{\partial \tau}\left(\frac{1}{2} \overline{\boldsymbol{u}^{\prime 2}}\right)=-S \overline{u_{x}^{\prime} u_{y}^{\prime}}-\nu \overline{\left|\nabla \times \boldsymbol{u}^{\prime}\right|^{2}} .
$$

Here the Reynolds stress $\overline{u_{x}^{\prime} u_{y}^{\prime}}$ provides an energy source for instability, which may be as large as we wish for sufficiently large $|S|$. It is well known (Joseph 1966), from this energy point of view, that the stability threshold (the smallest value of $|S|$ for stationary mean energy) is achieved by stream-wise vortices $\boldsymbol{u}^{\prime}=u_{x}^{\prime} \boldsymbol{e}_{x}+\boldsymbol{\nabla} \times\left(\chi^{\prime} \boldsymbol{e}_{x}\right)$ independent of $x$, where $\left[u_{x}^{\prime}, \chi^{\prime}\right]=\operatorname{Re}\left\{\left[\widehat{u}_{x}, \widehat{\chi}\right](y, \tau) \mathrm{e}^{\mathrm{i} \gamma z}\right\}$. These vortices (rolls with stream-function $\chi^{\prime}$ ) together with their streaks (aligned flow $u_{x}^{\prime}$ ) are also taken as the flow that will maximise the momentum transport between the planes in bounding theories of turbulence (Busse 1970).

Now, according to Squire's theorem (Squire 1933), the most unstable modes satisfying (6) are those that are wavelike in the $x$-direction $\left(\left[\boldsymbol{u}^{\prime}, p^{\prime}\right]=\operatorname{Re}\left\{[\widehat{\boldsymbol{u}}, \widehat{p}](y, \tau) \mathrm{e}^{\mathrm{i} \alpha x}\right\}\right)$. Unfortunately, whereas velocity gradients of the basic flow can provide a source of instability, their advective 
effects, as represented by the term $U \partial \boldsymbol{u}^{\prime} / \partial x$ which on average does no work (see (7)), is generally remarkably destructive owing to its kinematic effect. Indeed (6) admits no growing modes for any value of $S$, i.e. there is no stability threshold for any value of Re. To understand the nature of the problem we express (6) in the form

$$
\frac{\partial \widehat{\boldsymbol{u}}}{\partial \tau}+\mathrm{i} \alpha S y \widehat{\boldsymbol{u}}=-\mathrm{i} \alpha \widehat{\boldsymbol{p}} \boldsymbol{e}_{x}-\frac{\partial \widehat{p}}{\partial y} \boldsymbol{e}_{y}-S \widehat{u}_{y} \boldsymbol{e}_{x}+\nu\left(\frac{\partial^{2}}{\partial y^{2}}-\alpha^{2}\right) \widehat{\boldsymbol{u}} .
$$

Then if we consider the effect of advection alone, $\partial \widehat{\boldsymbol{u}} / \partial \tau+\mathrm{i} \alpha S y \widehat{\boldsymbol{u}}=\mathbf{0}$, on a cell-like structure proportional to $\exp \left(\mathrm{i} \frac{1}{2} \pi y / L\right)$ at $\tau=0$, we find that it evolves to

$$
\exp \left[-\mathrm{i}\left(S \alpha L \tau-\frac{1}{2} \pi\right) y / L\right],
$$

at time $\tau$. Whatever the value of $S$, whether positive or negative, the effective Reynolds number, measured by the size of $S \alpha L|\widehat{\boldsymbol{u}}| /\left|\nu \partial^{2} \widehat{\boldsymbol{u}} / \partial y^{2}\right|$, is of order $\operatorname{Re} /\left[\alpha L(S \tau)^{2}\right]$ and tends to zero as $\tau \uparrow \infty$. What happens is that the cell-like structure tips over (see, e.g., fig. 1 of Leprovost and Kim 2009) leading to an ever-decreasing length scale and enhanced dissipation.

Looked at from another point of view, we could associate with the disturbance a frequency

$$
\text { Say, }
$$

which varies (linearly) with position, a phenomenon well-known in plasma and astrophysics, where it is known to be destructive and called phase mixing. In particular Heyvaerts and Priest (1983) discuss the role of phase mixing in the heating of the solar corona, which is a topic that draws continuing interest; see, for example, De Moortel and Nakariakov (2012) particularly the paragraph containing eq. (4.1). Phase mixing due to the Keplerian motion in accretion discs provides a very direct link to Couette flow as demonstrated by Riols et al. (2013) and the numerous references therein. The operator $\partial / \partial \tau+\mathrm{i} S \alpha y$ in (8), or equivalently $\partial / \partial \tau+\mathrm{i} S y \partial / \partial x$ in (6a), lies at the heart of the connections to be made in this paper.

\subsection{Cylindrical Couette flow}

Steady cylindrical Couette flow (cCf) between two coaxial cylinders, of radii $R_{1}$ (inner) and $R_{2}$ (outer) and rotating at different angular velocities $\Omega_{1}$ and $\Omega_{2}(>0)$ (respectively), is perhaps more straightforward than $\mathrm{pCf}$ in the sense that at a sufficiently large rotation rate of the inner cylinder, as measured by the Taylor number Ta, the flow does becomes unstable. The presence of curvature, leading to rotation and the Coriolis acceleration associated with it, is crucial for the instability and leads to the possibility of axisymmetric rolls, called Taylor vortices (Taylor 1923), that enable angular momentum to be transferred outwards, as evinced by the azimuthal motion within the Taylor vortices and the increased stress on the rigid boundaries.

The existence of a critical Taylor number $\mathrm{Ta}_{\mathrm{cCf}}$ in $\mathrm{cCf}$ provides the basis upon which Davey (1962) developed a weakly nonlinear instability theory, valid when $0<\mathrm{Ta}-\mathrm{Ta}_{\mathrm{cCf}} \ll 1$, following methods pioneered by Stuart (1960) for plane Poiseuille flow. (Without such a critical Reynolds number in pCf this option is not available for analysing that flow.) In the case of narrow-gap cCf, for which the radius ratio

$$
\varepsilon \equiv\left(R_{2}-R_{1}\right) / R_{1},
$$

is small, Nagata (1990) considered the secondary bifurcations that follow from the finiteamplitude Taylor vortex state. He noted that his narrow-gap equations were equivalent to those for pCf when the inner cylinder counter-rotated sufficiently fast with $\Omega_{1}=-\Omega_{2}(<0)$. As well as the usual bifurcation to time-dependent non-axisymmetric wavy vortices, he found for sufficiently small $\Omega_{1}+\Omega_{2}$ that a bifurcation to non-axisymmetric steady modes occurred. 
By varying parameters (sometimes referred to as homotopy), he identified such steady finiteamplitude states for $\Omega_{1}+\Omega_{2}=0$. These provide steady solutions of the pCf-problem that are distinct from the classical pCf with its linear shear flow profile.

Nagata's study paved the way for a variety of other homotopy approaches. Clever and Busse (1992) took thermally driven convective rolls with their axes aligned to the applied shear, whereas Waleffe (2003) considered axial rolls maintained by an externally applied forcing. Waleffe considered the pCf-problem and artificially excited aligned vortices (rolls) with a stream function $\chi(y, z) \propto \cos (\pi y / 2 L) \sin \gamma z$ driven by a force with the transverse structure $\mathbf{F}=F_{A} \boldsymbol{\nabla} \times\left(\chi \boldsymbol{e}_{x}\right)$ for some constant $F_{A}$. The vortices transport linear momentum aligned with the applied flow, creating so-called streaks which are susceptible to inflexion point instabilities. Waleffe then investigated stability to wave-like disturbances proportional to $\mathrm{e}^{\mathrm{i} \alpha x}$ together with the ensuing finite amplitude steady motion described by a harmonic expansion involving

$$
\exp [\mathrm{i}(l \alpha x+n \gamma y)] \quad \begin{cases}n=0, \forall l & \text { for waves, } \\ l=0, \forall n & \text { for rolls (also streaks), }\end{cases}
$$

but also including all other integer pairs $l, n$ simultaneously non-zero. So, though both Nagata and Waleffe build upon underlying aligned vortices, Nagata's homotopy reduces $\Omega_{1}+\Omega_{2}$ to zero, while Waleffe reduces $F_{A}$ to zero instead, simultaneously adjusting boundary conditions on the flow as part of the homotopy. An interesting finding of Waleffe (2003) is a minimum value of $\operatorname{Re}=\operatorname{Re}_{\text {inf }}$ at some $\alpha=\alpha_{\text {inf }}, \gamma=\gamma_{\text {inf }}$, where such steady finite-amplitude motion occurs. Thereafter, for larger $\operatorname{Re}>\operatorname{Re}_{\text {inf }}$, a domain of solutions appears to exist in the $(\alpha, \gamma)-$ plane so extending the earlier results of Clever and Busse (1997). Waleffe also considered plane Poiseuille flow using the same techniques, but only later was his method used by Faisst and Eckhardt (2003) and Wedin and Kerswell (2004) to solve the corresponding pipe flow problem. The crucial idea behind the structure of the finite amplitude states both in pCf (Waleffe 2003) and circular Poiseuille flow (Wedin and Kerswell 2004), is that there is a mutual coupling of the stream-wise roll, their induced streaks and the wavelike disturbances, a mechanism that Waleffe (1997) describes as a self-sustaining process (SSP) (see also the overview by Eckhardt et al. 2007).

\subsection{Narrow-gap spherical Couette flow (sCf)}

As stream-wise rolls (i.e. Taylor vortices) arise naturally in narrow-gap cCf, there is no need to invoke a self-sustaining process when describing the finite-amplitude state close to the onset of instability. We contrast this situation with that of narrow-gap spherical Couette flow (sCf) between spheres, of radii $R_{1}$ and $R_{2}$, with $\varepsilon \ll 1$ (see (11)), with common centre and rotation axis but with different rotation rates $\Omega_{1}$ and $\Omega_{2}$. This configuration has attracted attention because it is amenable to asymptotic analysis. Building on the early studies of Walton (1978), Hocking and Skiepko (1981) and Hocking (1981), Soward and Jones (1983) resolved the linear stability problem which they characterised by a Reynolds number $\mathrm{Re}_{M}$ and an angular momentum ratio $\delta$ defined by

$$
\operatorname{Re}_{M} \equiv \frac{\varepsilon^{2}\left(R_{1}^{2} \Omega_{1}-R_{2}^{2} \Omega_{2}\right)}{\nu}, \quad \delta \equiv \frac{R_{1}^{2} \Omega_{1}-R_{2}^{2} \Omega_{2}}{R_{1}^{2} \Omega_{1}+R_{2}^{2} \Omega_{2}},
$$

respectively, and a Taylor number

$$
\mathrm{Ta} \equiv\left[\frac{2\left(R_{2}-R_{1}\right)}{R_{2}+R_{1}}\right]^{3} \frac{\left(R_{1}^{2} \Omega_{1}\right)^{2}-\left(R_{2}^{2} \Omega_{2}\right)^{2}}{\nu^{2}}=\left[\frac{2 R_{1}}{R_{2}+R_{1}}\right]^{3} \frac{\operatorname{Re}_{M}^{2}}{\varepsilon \delta} .
$$

Two cases are of particular interest to us: when the outer sphere is at rest, $\Omega_{2}=0$, for which $\delta=1$; and the almost co-rotation of the spheres when $\Omega_{2} \approx \Omega_{1}$ or, more precisely, $0<\delta \ll 1$. 
In both instances the onset of instability is characterised by $\mathrm{Ta}=\mathrm{Ta}_{\mathrm{sCf}}=\mathrm{O}(1)$ so that by (13c) the Reynolds number $\operatorname{Re}_{M}=\mathrm{O}\left((\varepsilon \delta)^{1 / 2}\right) \ll 1$.

The essential idea is that any instability is manifested by axisymmetric Taylor vortices localised near the equator, where their driving by the centrifugal force is maximised. Each Taylor vortex occurs on a short latitudinal length scale which suggests an expansion procedure based on the marginal solution for the onset of instability for rotating cylinders (cCf), for which separable solutions for the radial velocity perturbation may be expressed as

$$
W(\varpi) \exp \left[\mathrm{i}\left(\varepsilon^{-1} \breve{k} \theta-\breve{\omega} \tau\right)\right]+\text { c.c.. }
$$

Here $\tau$ denotes the time and c.c. the complex conjugate, for some critical Taylor number $\mathrm{Ta}_{c}=\mathrm{Ta}_{\mathrm{CCf}}$, critical wave number $\breve{k}_{c}=\breve{k}_{\mathrm{cCf}}$ and frequency $\breve{\omega}_{c}=\breve{\omega}_{\mathrm{CCf}}=0$, namely the local values determined by the cCf-problem. Though the primary basic flow, on which the smallscale Taylor vortices ride, is azimuthal, there is a secondary large-scale meridional circulation consisting of two giant cells in each hemispherical shell $-\frac{1}{2} \pi<\theta<0$ and $0<\theta<\frac{1}{2} \pi$. The sense of the circulation is such that motion close to the inner sphere is directed towards the equatorial plane, thrown outwards at the equator and then returned poleward near the outer sphere. Though this secondary circulation is smaller than the primary azimuthal flow by a factor $\mathrm{O}\left(\mathrm{Re}_{M}\right)$, its radial asymmetry, of magnitude proportional to $\delta$, is responsible for the phase mixing identified by the term ixa in (1) (but see also (4a)) leading to important ramifications for both stability and the subsequent nonlinear development. Soward and Jones (1983) showed that the true critical Taylor number $\mathrm{Ta}_{\mathrm{sCf}}$ exceeds $\mathrm{Ta}_{c}\left(=\mathrm{Ta}_{\mathrm{cCf}}\right)$ by an amount

$$
\mathrm{Ta}_{\mathrm{sCf}}-\mathrm{Ta}_{c}=\mathrm{O}\left(\delta^{2}\right),
$$

independent of $\varepsilon$, which therefore remains finite as $\varepsilon \downarrow 0$.

This finding motivates much of that which follows. Our point of view is that the value of $\mathrm{Ta}_{\mathrm{sCf}}$ is really an irrelevance for the nature of any finite amplitude motion in the limit $\varepsilon \downarrow 0$, so that rather than expanding about the true linear instability point $\mathrm{Ta}_{\mathrm{sCf}}$, we expand about the smaller value $\mathrm{Ta}_{c}\left(=\mathrm{Ta}_{\mathrm{cCf}}\right)$, using the cCf-results to provide our basic approximation. This is the basis on which we construct our complex Ginzburg-Landau equation (CGLe) (20) in the coming section. Though unusual, Soward (1977) employed exactly this strategy to construct and solve the amplitude equations for a thermal convection problem in a self-gravitating rotating sphere. However, in our narrow-gap sCf-case, the zeroth-order approximation of (20), namely (1), encounters under linearisation the same difficulty faced by the linear pCf-problem. Specifically there is no linear instability threshold for the lowest-order problem about which we are expanding. This is the most significant feature in common with pCf. We emphasise that, because of the small parameter $\varepsilon$, the situation for narrow-gap $\mathrm{sCf}$ is different to other problems such as plane Poiseuille flow, for which finite amplitude disturbances persist at Reynolds numbers $\mathrm{Re}$ below the critical value $\mathrm{Re}_{c}$ (say). In narrow-gap sCf the relevant parameter is $\left(\mathrm{Ta}_{\mathrm{sCf}}-\mathrm{Ta}\right) / \varepsilon^{2 / 3}=\mathrm{O}\left(\varepsilon^{-2 / 3}\right)$ which for the cases of interest tends to infinity as $\varepsilon \downarrow 0$. By contrast, in plane Poiseuille flow the relevant parameter is $\operatorname{Re}_{c}-\operatorname{Re}=\mathrm{O}(1)$, a finite number.

\section{A complex Ginzburg-Landau equation (CGLe)}

The cCf-solution (14) provides the starting point for the solution of the narrow-gap sCfproblem for $|\theta|$ small in the neighbourhood of the equatorial plane. On the basis that the variations of the eigenfunction with $\theta$ can be ignored, we envisage that the frequency satisfies a local dispersion relation of the form

$$
\breve{\omega}=\breve{\omega}(\theta, \breve{k} ; \mathrm{Ta})
$$


valid for the assumed $W(\varpi)$ in (14) provided that $|\theta|,\left|\breve{k}-\breve{k}_{c}\right|$ and $\breve{\omega}$ are all acceptably small. The symmetry of the basic flow implies that $\breve{\omega}$ has the property

$$
\breve{\omega}^{*}(\theta, \breve{k} ; \mathrm{Ta})=-\breve{\omega}(-\theta, \breve{k} ; \mathrm{Ta}),
$$

where the superscript ${ }^{*}$ denotes complex conjugate. At critical, both the frequency and group velocities vanish

$$
\breve{\omega}_{c}=0,\left.\quad \quad \breve{\omega}_{, \breve{k}}\right|_{c}=0,
$$

in which we have introduced the standard partial derivative notation of subscript comma (e.g., here $\breve{\omega}_{, \breve{k}} \equiv \partial \breve{\omega} / \partial \breve{k}$ ), while following the convention advocated in section 1 the subscript $c$ denotes values at $\left(0, \breve{k}_{c} ; \mathrm{Ta}_{c}\right)$. With account taken of the properties $(17 \mathrm{a}, \mathrm{b})$, the Taylor series expansion of the dispersion relation about the cCf-critical gives

$$
-\mathrm{i} \widetilde{\omega}=-\left.\mathrm{i} \breve{\omega}_{, T a}\right|_{c} \widetilde{T}-\left.\mathrm{i} \breve{\omega}_{, \theta}\right|_{c} \theta-\left.\frac{1}{2} \mathrm{i} \breve{\omega}_{, \theta \theta}\right|_{c} \theta^{2}-\left.\breve{\omega}_{, \theta \breve{k}}\right|_{c} \theta(\mathrm{i} \widetilde{k})+\left.\frac{1}{2} \mathrm{i} \breve{\omega}_{, \breve{k} \breve{k}}\right|_{c}(\mathrm{i} \widetilde{k})^{2}+\cdots,
$$

where

$$
\widetilde{k}=\breve{k}-\breve{k}_{c}, \quad \widetilde{\omega}=\breve{\omega}-\breve{\omega}_{c} \quad \text { and } \quad \widetilde{T}=\mathrm{Ta}-\mathrm{Ta}_{c} .
$$

It means that, if we consider the modulation of the Taylor vortices characterised by

$$
\widetilde{a}(\theta, \tau) W(\varpi) \exp \left(\mathrm{i} \varepsilon^{-1} \breve{k}_{c} \theta\right)+\text { c.c. },
$$

their amplitude $\widetilde{a}(\theta, \tau)$ satisfies the CGLe

$$
\frac{\partial \widetilde{a}}{\partial \tau}+\left.\breve{\omega}_{, \theta \breve{k}}\right|_{c} \theta \varepsilon \frac{\partial \widetilde{a}}{\partial \theta}=\left(-\left.\mathrm{i} \breve{\omega}_{, T a}\right|_{c} \widetilde{T}-\left.\mathrm{i} \breve{\omega}_{, \theta}\right|_{c} \theta-\left.\frac{1}{2} \mathrm{i} \breve{\omega}_{, \theta \theta}\right|_{c} \theta^{2}\right) \widetilde{a}+\frac{1}{2} \mathrm{i} \breve{\omega},\left.\breve{k} \breve{k}\right|_{c} \varepsilon^{2} \frac{\partial^{2} \widetilde{a}}{\partial \theta^{2}}-\mathcal{C}|\widetilde{a}|^{2} \widetilde{a}
$$

Here we have included the nonlinear Stuart-Landau term $\mathcal{C}|\widetilde{a}|^{2} \widetilde{a}$ (Stuart 1960), derived by Davey (1962) in the cCf-context that applies here, in the anticipation that we wish to find weakly nonlinear finite-amplitude states. Consistent with the symmetry property (16b) both $\left.\breve{\omega}_{, \theta}\right|_{c}$ and $\left.i \breve{\omega}_{, \theta \theta}\right|_{c}$ are real at $\theta=0$ and furthermore, in view of $\left.\breve{\omega}_{, \breve{k}}\right|_{c}=0$ (see $(17 \mathrm{~b})$ ), $\left.\breve{\omega}_{, \theta \breve{k}}\right|_{c}$ is real too. In fact, numerical solutions of the eigenvalue problem for $W(\varpi)$ generally show that

$$
\left.\breve{\omega}_{, \theta}\right|_{c}<0,\left.\quad \quad \breve{\omega}_{, \theta \breve{k}}\right|_{c}<0,\left.\quad \quad \mathrm{i} \breve{\omega}_{, \theta \theta}\right|_{c}>0 .
$$

The coefficients $-\mathrm{i} \breve{\omega},\left.\mathrm{Ta}\right|_{c}, i \breve{\omega},\left.\breve{k} \breve{k}\right|_{c}$ and $\mathcal{C}$ also turn out to be real and positive:

$$
-\mathrm{i} \breve{\omega},\left.\mathrm{Ta}\right|_{c}>0, \quad \mathrm{i} \breve{\omega},\left.\breve{k} \breve{k}\right|_{c}>0, \quad \mathcal{C}>0 .
$$

Each of the terms in (20) has a physical interpretation relative to the rate term $\partial \widetilde{a} / \partial \tau$. The term $\left.\breve{\omega}_{, \theta \breve{k}}\right|_{c} \theta \varepsilon \partial \widetilde{a} / \partial \theta$ describes advection at the group velocity $\left.\varepsilon \breve{\omega}_{, \theta \breve{k}}\right|_{c} \theta$ towards the equator, but increasing in magnitude linearly with angular distance $|\theta|$ away from it. The term $\left(-\mathrm{i} \breve{\omega},\left.T a\right|_{c} \widetilde{T}-\frac{1}{2} \mathrm{i} \breve{\omega},\left.\theta \theta\right|_{c} \theta^{2}\right) a$ drives the instability provided

$$
|\theta|<\theta_{u s}, \quad \quad \quad \text { where } \quad \theta_{u s}=\left[-\left.\mathrm{i} \breve{\omega}_{, T a}\right|_{c} \widetilde{T} /\left.\frac{1}{2} \mathrm{i} \breve{\omega}_{, \theta \theta}\right|_{c}\right]^{1 / 2},
$$

implying that the range $-\theta_{u s}<\theta<\theta_{u s}$ defines the potentially unstable region for infinitesimal disturbances. The term $\frac{1}{2} \varepsilon^{2} \mathrm{i} \breve{\omega},\left.\breve{k} \breve{k}\right|_{c} \partial^{2} \widetilde{a} / \partial \theta^{2}$ describes diffusion, while the Stuart-Landau term $-\mathcal{C}|\widetilde{a}|^{2} \widetilde{a}$ provides nonlinear quenching. All these competing effects are well understood.

The phase mixing term $-\left.\mathrm{i} \breve{\omega}_{, \theta}\right|_{c} \theta \widetilde{a}$ within (20) leads to the temporally reducing length scale effect, which operates in the manner described at the start of the Introduction as it pertained to linear disturbances in pCf governed by (8) and quantified by (9). Though there are obvious differences in how the effects originate - in pCf it is normal to the boundaries while in $\mathrm{sCF}$ it is transverse to them - the stabilising influence and its consequences involve ingenuity by 
the physical system to overcome them. Of crucial importance to what follows is the angular length scale

$$
\ell_{p m}=\left[-\left.\frac{1}{2} \mathrm{i} \breve{\omega}_{\breve{k} \breve{k}}\right|_{c} /\left.\breve{\omega}_{, \theta}\right|_{c}\right]^{1 / 3} \varepsilon^{2 / 3},
$$

on which the phase mixing and diffusion terms balance.

\subsection{Non-dimensionalisation on the length scale $\ell_{p m}$}

In order to expose the importance of the length $\ell_{p m}$, we further non-dimensionalise as follows:

$$
\begin{aligned}
\theta & =\ell_{p m} \mathrm{x}, & \widetilde{a} & =\frac{\left(\left.\frac{1}{2} \mathrm{i} \breve{\omega}_{\breve{k} \breve{k}}\right|_{c} / \mathcal{C}\right)^{1 / 2}}{\ell_{p m} / \varepsilon} \mathrm{a}, \\
\tau & =\frac{\left(\ell_{p m} / \varepsilon\right)^{2}}{\left.\frac{1}{2} \mathrm{i} \breve{\omega}_{\breve{k} \breve{k}}\right|_{c}} t, & \widetilde{T} & =\frac{\left.\frac{1}{2} \mathrm{i} \breve{\omega}_{\breve{k} \breve{k}}\right|_{c}}{-\left.\mathrm{i} \breve{\omega}_{, \mathrm{Ta}}\right|_{c}} \frac{\mathrm{T}}{\left(\ell_{p m} / \varepsilon\right)^{2}},
\end{aligned}
$$

so that (20) becomes

$$
\frac{\partial a}{\partial t}-D{ }_{x} \frac{\partial a}{\partial x}=\left(T+i x-\left(\Upsilon_{x}\right)^{2}\right) a+\frac{\partial^{2} a}{\partial x^{2}}-|a|^{2} a,
$$

in which the remaining independent (real) parameters $\mathrm{D}(>0)$ and $\Upsilon(>0)$ are

$$
\mathrm{D}=\frac{-\left.2 \breve{\omega}_{, \theta \breve{k}}\right|_{c}}{\left[-\left.\left.\breve{\omega}_{, \theta \theta}\right|_{c} \breve{\omega}_{\breve{k} \breve{k}}\right|_{c}\right]^{1 / 2}} \quad \text { and } \quad \Upsilon=\left[\frac{\left.\breve{\omega}_{, \theta \theta}\right|_{c}}{\left.\breve{\omega}_{\breve{k} \breve{k}}\right|_{c}}\right]^{1 / 2} \ell_{S C}^{2} / \varepsilon
$$

We henceforth refer to (25a) as the full CGLe.

It is worth noting immediately in order to avoid confusion later that, following the length and time scale changes $(24 \mathrm{a}, \mathrm{c})$, local travelling wave solutions of $(25 \mathrm{a})$, expressed in the form

$$
\mathrm{a}(\mathrm{x}, t) \propto \exp [\mathrm{i}(\mathrm{kx}+\omega t)]
$$

themselves correspond to the perturbation wave number and frequency (see (18b,c)) changes

$$
\left(\ell_{p m} \varepsilon\right) \widetilde{k}=\mathrm{k}, \quad \frac{\left(\ell_{p m} / \varepsilon\right)^{2}}{\frac{1}{2} \mathrm{i} \breve{\omega}_{\left.\breve{k} \breve{k}\right|_{c}}} \widetilde{\omega}=-\omega .
$$

Since $\left.\breve{\omega}_{, \theta}\right|_{c}<0$ (see (21a)), we have for convenience introduced a sign change for the frequency, that should not be overlooked because we will use this reversed sign throughout in what follows.

The boundary conditions appropriate to localised solutions are

$$
\mathrm{a} \rightarrow 0 \quad \text { as } \quad \mathrm{x} \rightarrow \pm \infty .
$$

Note that for any solution $\mathrm{a}(\mathrm{x}, t)$ of $(25 \mathrm{a})$, there is a second solution obtained by changing the sign of $x$ (reflection) and taking the complex conjugate:

$$
\mathrm{a}^{*}(-\mathrm{x}, t),
$$

an operation that we refer to as complex conjugate reflection (c.c.r.). If

$$
\mathrm{e}^{-\mathrm{i} \varphi} \mathrm{a}(\mathrm{x}, t)=\mathrm{e}^{\mathrm{i} \varphi} \mathrm{a}^{*}(-\mathrm{x}, t), \quad \text { (SP-solution) }
$$

for some constant phase angle $\varphi$, we say that the solution is symmetry preserved (SP), otherwise we say it is symmetry broken (SB). 


\begin{tabular}{|c|c|c|c|c|c|}
\cline { 2 - 6 } \multicolumn{1}{c|}{} & \multicolumn{3}{c|}{ Lengths } & \multicolumn{2}{c|}{ Parameters } \\
\hline Ranges & $\ell_{c}$ & $\ell_{p m}$ & $\theta_{u s}$ & $\mathrm{D}$ & $\Upsilon$ \\
\hline $\mathrm{O}\left(\varepsilon^{1 / 2}\right)<\delta<\mathrm{O}(1)$ & $\varepsilon$ & $\varepsilon^{2 / 3} / \delta^{1 / 3}$ & $(\varepsilon \delta)^{1 / 3}$ & $\delta^{1 / 2}$ & $\varepsilon^{1 / 3} / \delta^{2 / 3}$ \\
$\delta=\mathrm{O}\left(\varepsilon^{1 / 2}\right)$ & $\varepsilon$ & $\varepsilon^{1 / 2}$ & $\varepsilon^{1 / 2}$ & $\varepsilon^{1 / 4}$ & 1 \\
$\delta=\mathrm{O}(1)$ & $\varepsilon$ & $\varepsilon^{2 / 3}$ & $\varepsilon^{1 / 3}$ & 1 & $\varepsilon^{1 / 3}$ \\
\hline
\end{tabular}

Table 1. Order of magnitude estimates. The relative sizes of the Taylor vortex width $\ell_{c}$, the pulse width $\ell_{p m}$ and the extent of the unstable domain $\theta_{u s}$ together with parameters $\mathrm{D}$ and $\Upsilon$ in the range $\mathrm{O}\left(\varepsilon^{1 / 2}\right) \leq \delta \leq \mathrm{O}(1)$.

\subsection{A time-harmonic expansion}

By introducing the amplitude factor $\exp (\mathrm{i} \Omega t)$ with the drifting phase $\Omega t$ so that $\mathrm{a}(\mathrm{x}, t)=$ $\mathrm{e}^{\mathrm{i} \Omega t} \mathrm{~b}(\mathrm{x}, t)$ where $\Omega$ is a constant, it follows from (25a) that $\mathrm{b}$ satisfies

$$
\frac{\partial \mathrm{b}}{\partial t}-\mathrm{D} \Upsilon \times \frac{\partial \mathrm{b}}{\partial \mathrm{x}}=\left(\mathrm{T}+\mathrm{i}(\mathrm{x}-\Omega)-(\Upsilon \times \mathrm{x})^{2}\right) \mathrm{b}+\frac{\partial^{2} \mathrm{~b}}{\partial \mathrm{x}^{2}}-|\mathrm{b}|^{2} \mathrm{~b} .
$$

When a steady solution $b=b(x)$ exists, it generates two distinct Hocking and Skiepko (1981) solutions

$$
\mathrm{a}(\mathrm{x}, t)=\mathrm{e}^{\mathrm{i} \Omega t} \mathrm{~b}(\mathrm{x}) \quad \text { and its c.c.r. } \quad \mathrm{e}^{-\mathrm{i} \Omega t} \mathrm{~b}^{*}(-\mathrm{x}) .
$$

With $\Omega \neq 0$ they cannot satisfy the SP-condition (30) for all time $t$ and so must be SB-modes.

An important class of unsteady solutions turns out to have the form

$$
\mathrm{b}(\mathrm{x}, t)=\sum_{l=-\infty}^{\infty} \mathrm{b}_{l}(\mathrm{x}) \exp \left[\mathrm{i}\left(l+\frac{1}{2}\right) \omega t\right]
$$

where $b_{l}$ (bounded as $x \rightarrow \pm \infty$ ) satisfies

$$
\begin{aligned}
\mathrm{i}\left(l+\frac{1}{2}\right) \mathrm{b}_{l}-\mathrm{D} \Upsilon \times \frac{\partial \mathrm{b}_{l}}{\partial \mathrm{x}}=\left(\mathrm{T}+\mathrm{i}(\mathrm{x}-\Omega)-(\Upsilon \mathrm{x})^{2}\right) \mathrm{b}_{l}+\frac{\partial^{2} \mathrm{~b}_{l}}{\partial \mathrm{x}^{2}} \\
-\sum_{m=-\infty}^{\infty} \sum_{n=-\infty}^{\infty} \mathrm{b}_{m} \mathrm{~b}_{m+n-l}^{*} \mathrm{~b}_{n}
\end{aligned}
$$

Solutions of the form (33a) are SP when

$$
\mathrm{b}_{l-1}(\mathrm{x})=\mathrm{b}_{-l}^{*}(-\mathrm{x}) \quad \forall n \quad \text { and } \quad \Omega=0 .
$$

\subsection{The case $\mathrm{D} \ll 1, \Upsilon=\mathrm{O}(1)$}

Our problem has been set up on the basis that $\varepsilon \ll 1$ and $0<\delta \leq 1$, where $\delta=1$ corresponds to a stationary outer sphere and $0<\delta \ll 1$ corresponds to almost co-rotation of the two spheres. Over that small- $\delta$ range all the derivatives of $\breve{\omega}$ are essentially $\mathrm{O}(1)$ with the exception of $\left.\breve{\omega}_{, \theta}\right|_{c}$ and $\left.\breve{\omega}_{, \theta \breve{k}}\right|_{c}$, which are both $\mathrm{O}(\delta)$, see Soward and Jones (1983). This implies that

$$
\ell_{p m}=\mathrm{O}\left(\varepsilon^{2 / 3} / \delta^{1 / 3}\right)
$$

with the consequences

$$
\mathrm{D}=\mathrm{O}\left(\delta^{1 / 2}\right) \quad \text { and } \quad \Upsilon=\mathrm{O}\left(\varepsilon^{1 / 3} / \delta^{2 / 3}\right) .
$$

If we identify the almost co-rotation limit with $\delta=\mathrm{O}\left(\varepsilon^{1 / 2}\right)$, for which $\Upsilon=\mathrm{O}(1)$ and $\mathrm{D}=\mathrm{O}\left(\varepsilon^{1 / 4}\right)$, all the terms in (25a) are then important for $\mathrm{x}=\mathrm{O}(1)$ except for $-\mathrm{D} \Upsilon \mathrm{x} \partial \mathrm{a} / \partial \mathrm{x}$. Significantly the two controlling length scales $\ell_{p m}$ and $\theta_{u s}$ are both the same size $\mathrm{O}\left(\varepsilon^{1 / 2}\right)$, but remain large compared to the Taylor vortex width $\ell_{c}=\mathrm{O}(\varepsilon)$ (see table 1 ). 


\subsection{The case $\mathrm{D}=\mathrm{O}(1), \Upsilon \ll 1$}

The problem takes on an added level of complexity in the physically more relevant parameter range $\varepsilon^{1 / 2} \ll \delta \leq 1$, because then our full CGLe (25a) continues to contain an important small parameter $\Upsilon$. Since the potentially unstable range is large $\mathrm{x}_{u s} \gg 1$ (equivalently $\theta_{u s} \gg \ell_{p m}$, see table 1), a multiple length scale problem ensues for which the leading-order balance is dominated by phase mixing: $\partial \mathrm{a} / \partial t=i \times \mathrm{xa}$ for large $\mathrm{x}$ with solutions

$$
\mathrm{a} \propto \exp (\mathrm{ixt}) \quad \text { for } \quad 1 \ll \mathrm{x}=\mathrm{O}\left(\Upsilon^{-1}\right) .
$$

We describe two possible systematic approaches to this multiple scale problem. Both involve setting up solutions on the intermediate length scale $\ell_{p m}$ relative to an origin $\mathrm{x}=\Upsilon^{-1} \mathrm{X}$ somewhere on the long length scale $\theta_{\text {us }}$.

In the first approach we take a fixed origin $\mathrm{x}=\mathrm{x}_{0}$, a constant, and consider local solutions

$$
\mathrm{a}(\mathrm{x}, t)=\mathrm{e}^{\mathrm{i} \mathrm{x}_{0} t} b(x, t), \quad \text { where } \quad\left\{\begin{array}{l}
\mathrm{x}=\mathrm{x}_{0}+x, \\
\mathrm{x}_{0}=\Upsilon^{-1} \mathrm{x}_{0},
\end{array}\right.
$$

similar to (32) with $\Omega=\mathrm{x}_{0}$. Relative to the shifted origin the CGLe (31) may be approximated to lowest order by

$$
\frac{\partial b}{\partial t}-\mathrm{DX}_{0} \frac{\partial b}{\partial x}=(\lambda+\mathrm{i} x) b+\frac{\partial^{2} b}{\partial x^{2}}-|b|^{2} b \quad\left(\lambda=\mathrm{T}-\mathrm{X}_{0}^{2}\right),
$$

for $x=\mathrm{O}(1)$. This form has the advantage that it is compatible with the discrete frequency description (33a) but has the drawback that rather than having simply the one parameter $\lambda$ to adjust, it involves the additional parameter $\mathrm{DX}_{0}$. This is an added level of complexity which we can do without.

An alternative strategy, which appears to be sufficient to meet our objectives, is to use a non-stationary origin $\mathrm{x}=\Upsilon^{-1} \mathrm{X}(t)$, moving with the group velocity $-\mathrm{D} \Upsilon \mathrm{x}=-\mathrm{DX}(t)$. We therefore write

$$
\mathbf{x}=\Upsilon^{-1} \mathbf{X}(t)+x, \quad \text { where } \quad \mathbf{X}(t)=\mathbf{X}_{0} \exp [-\mathrm{D} \Upsilon t],
$$

and $\mathrm{x}_{0}=\Upsilon^{-1} \mathrm{x}_{0}$ is the initial position of the origin at $t=0$. Then we make the change of variables

$$
\mathrm{a}(\mathrm{x}, t)=a(x, t) \exp \left[\mathrm{i} \Upsilon^{-1} \psi(t)\right], \quad \text { where } \quad \psi(t)=(\mathrm{D} \Upsilon)^{-1}\left[\mathrm{X}_{0}-\mathrm{X}(t)\right],
$$

so that

$$
\mathrm{a}=a(x, t) \exp \left[\mathrm{i} \Upsilon^{-1} \mathbf{X}_{0}\left(t-\frac{1}{2} \mathrm{D} \Upsilon t^{2}+\cdots\right)\right] .
$$

Here the dominant contribution $\mathrm{a}=a(x, t) \exp \left(\mathrm{i} \Upsilon^{-1} \mathbf{X}_{0} t\right)$ is evidently consistent with (38a). Significantly the complete representation (40a-d) ensures that not only is the dominant phase mixing contribution $i \Upsilon^{-1} \mathrm{X}_{0}$ a removed from the right-hand side of the CGLe (25a) at lowest order (as it is in (39)), but also the dominant part $-\mathrm{DX}_{0} \partial \mathrm{a} / \partial \mathrm{x}$ of the group velocity term remaining on the left-hand side of (39) is removed from (25a) at the next order. The new amplitude $a(x, t)$ then satisfies the exact equation

$$
\frac{\partial a}{\partial t}-\mathrm{D} \Upsilon x \frac{\partial a}{\partial x}=(\Lambda(\mathrm{X}, x)+\mathrm{i} x)+\frac{\partial^{2} a}{\partial x^{2}}-|a|^{2} a
$$

where

$$
\Lambda(\mathrm{X}, x)=\mathrm{T}-(\mathrm{X}(t)+\Upsilon x)^{2}
$$

So, when

$$
\Upsilon \ll 1
$$


and subject to the limited restrictions

$$
x \ll \Upsilon^{-1} \quad \text { for } \quad t \ll \Upsilon^{-1},
$$

on the space and time regimes, the group velocity term -D $x x \partial a / \partial x$ in (41a) may be neglected and $\Lambda$ in (41b) may be approximated by the constant

$$
\lambda=\Lambda\left(\mathrm{X}_{0}, 0\right)=\mathrm{T}-\mathrm{X}_{0}^{2} \leq \mathrm{T} .
$$

Under those conditions $a(x, t)$ solves $\partial a / \partial t=(\lambda+\mathrm{i} x) a+\partial^{2} a / \partial x^{2}-|a|^{2} a$, namely (1) above, which differs from (39) only by the removal of the term $-\mathrm{DX}_{0} \partial b / \partial x$ on its left-hand side.

The failure of the solution (40c) to hold on both the long length $x=\mathrm{O}\left(\Upsilon^{-1}\right)$ and long time $t=\mathrm{O}\left(\Upsilon^{-1}\right)$ is potentially serious as these non-uniformities prevent the solutions holding everywhere in space and persisting indefinitely in time. Moreover, they are not compatible with the temporally periodic formulation (33a), but the numerical evidence suggests that the drifting style (40c) is more appropriate. This means that the solutions of (1) should not be regarded as true solutions of (25a), but rather any space-time periodic solution of (1) simply approximates the true solution for a limited space-time interval. The realised solution composed of these building blocks is likely to be weakly chaotic in both space and time (cf. other pattern selection problems like the occurrence of roll dislocations in Bénard convection in thin layers of large horizontal extent).

\subsection{The homotopy from $\mathrm{D} \ll 1, \Upsilon=\mathrm{O}(1)$ towards the case $\mathrm{D}=\mathrm{O}(1), \Upsilon \ll 1$}

Despite the apparent simplicity of the equation, solutions of (1) bounded as $x$ tends to both $\pm \infty$ possess a surprisingly rich structure which originates from the phase mixing term ixa. The challenge for its solution comes about because, just like the classic pCf-problem, there is no critical value of $\lambda$ at which the trivial solution $a=0$ bifurcates to a finite-amplitude state. So, following the strategy adopted for the pCf-case, to find $a \neq 0$ solutions of (1) which remain finite as $t \uparrow \infty$ we are led to use homotopy methods that involve the inclusion of extra terms in the CGLe. Provided the extended CGLe possesses an instability threshold, the subsequent bifurcation sequence can then be studied. The hope is that finite-amplitude solutions can be identified that persist after the extra terms are dropped. The natural way to do that is to investigate the (near co-rotation) case $\delta=\mathrm{O}\left(\varepsilon^{1 / 2}\right)$, for which $\mathrm{D}=\mathrm{O}\left(\varepsilon^{1 / 4}\right)$ and $\Upsilon=\mathrm{O}(1)$ (see table 1$)$, and then solve $(25 \mathrm{a})$ for $\mathrm{x}=\mathrm{O}(1)$ with the term $-\mathrm{D} \Upsilon \times \mathrm{x} \partial \mathrm{a} / \partial \mathrm{x}$ neglected but the term $-(\Upsilon \times)^{2}$ a retained:

$$
\frac{\partial \mathrm{a}}{\partial t}=\left(\mathrm{T}+\mathrm{ix}-(\Upsilon \mathrm{X})^{2}\right) \mathrm{a}+\frac{\partial^{2} \mathrm{a}}{\partial \mathrm{x}^{2}}-|\mathrm{a}|^{2} \mathrm{a} .
$$

The homotopy then consists of decreasing the value of $\Upsilon$ with the purpose of reaching an understanding of the solutions of (1) which pertain to the limit $\Upsilon \downarrow 0$.

\section{The solution structure of the full CGLe (25a)}

\subsection{The homotopy: Almost co-rotation $\mathrm{O}\left(\varepsilon^{1 / 2}\right) \leq \delta \ll 1$}

In the almost co-rotation limit $\delta=\mathrm{O}\left(\varepsilon^{1 / 2}\right)$, the eigenvalue problem posed by the steady solutions of (45) satisfying the linearised equation (i.e., with the term $|a|^{2}$ a neglected) subject to the boundary condition $\mathrm{a} \rightarrow 0$ as $|\mathrm{x}| \rightarrow \infty$ leads to a discrete spectrum whose lowest mode is

$$
\mathrm{a}=\mathrm{a}_{0} \exp \left[-\frac{1}{2} \Upsilon \mathrm{x}^{2}+\mathrm{i} \frac{1}{2} \Upsilon^{-1} \mathrm{x}\right] \quad \text { with } \quad \mathrm{T}=\frac{1}{4} \Upsilon^{-2}+\Upsilon
$$




\begin{tabular}{|c||c|r|c|c|}
\hline $\mathrm{T}$ & $\mathrm{a}_{\max }$ & $M$ & $\omega$ & $\mathrm{x}_{0}$ \\
\hline 3.375 & 1.390 & 46 & 2.313 & $?$ \\
3.875 & 1.605 & 6 & 2.423 & 0.578 \\
$\mathrm{SP} 3.975$ & 1.431 & 10 & 2.454 & 1.227 \\
4.0 & 1.624 & 9 & 2.437 & 0.409 \\
4.5 & 1.791 & 67 & 2.493 & 0.205 \\
\hline
\end{tabular}

Table 2. The characteristics of the SB-periodic solutions (50) found by Harris et al. (2003), when $\Upsilon=\frac{1}{4}$ taken from their Table 1 under the parameter scalings $\kappa \mapsto \frac{1}{2} \Upsilon^{-3 / 2}=4, \lambda \mapsto 4 \mathrm{~T}, a_{\max } \mapsto 2 \mathrm{a}_{\max },(\Omega, \omega) \mapsto 4\left(\mathrm{x}_{0}, \omega\right)$ (see also figs. 21-23 of Harris et al. (2003) for $\mathrm{T} \approx 3.875,4.5,3.375$ ). No reliable value of $\mathrm{x}_{0}$ for $\mathrm{T} \approx 3.375$ could be discerned (see Harris et al. 2003). The solution for $\mathrm{T} \approx 3.975$ marked by SP in the table is the symmetry preserving solution illustrated in fig. 20 of Harris et al. (2003).

where $\mathrm{a}_{0}$ is a constant. The complete spectrum is

$$
\mathrm{T}=\mathrm{T}_{n}=\frac{1}{4} \Upsilon^{-2}+(2 n+1) \Upsilon \quad(n=0,1,2,3, \cdots),
$$

which determine modes of SP-type (see (30)) like (46a). Interestingly for small $\Upsilon$ (i.e., $\delta \gg$ $\left.\varepsilon^{1 / 2}\right)$, the lowest mode $\mathrm{T}=\mathrm{T}_{0}$ describes a wave of short wavelength $4 \pi \Upsilon$ modulated by a Gaussian of large width $\mathrm{O}\left(\Upsilon^{-1 / 2}\right)$. More significantly, (46b) implies $\mathrm{T}=\mathrm{O}\left(\Upsilon^{-2}\right)$ with the consequence noted by Soward and Jones (1983) that at the onset of stability

$$
\begin{aligned}
\mathrm{Ta}_{\mathrm{sCf}}-\mathrm{Ta}_{c} & =\widetilde{T}_{\mathrm{sCf}}=\mathrm{O}\left(\mathrm{T}_{0} \varepsilon^{2} / \ell_{p m}^{2}\right) \\
& =\mathrm{O}\left(\Upsilon^{-2} \varepsilon^{2} / \ell_{p m}^{2}\right)=\mathrm{O}\left(\delta^{2}\right) \quad \text { for } \quad \mathrm{O}\left(\varepsilon^{1 / 2}\right) \leq \delta \ll 1,
\end{aligned}
$$

(see (18d), (24d) and table 1) independent of the value of $\varepsilon$. The divergence of $\mathrm{T}_{0}$ with decreasing $\Upsilon$ alerts us to the fact that the theory breaks down for $\delta=\mathrm{O}(1)$; the correct development for that case was given by Soward and Jones (1983). Fortunately we do not need to explore that story here, because the bifurcation at $\lambda=\mathrm{O}\left(\Upsilon^{-2}\right)$ moves to infinity as $\Upsilon \downarrow 0$, where it becomes an irrelevance not only because it has moved far away but because the amplitude of the bifurcated solutions tend to zero as well (see (49) below).

Harris et al. (2000) considered the weakly nonlinear theory that follows the bifurcation at $\mathrm{T}=\mathrm{T}_{0}$ and found that a finite amplitude SP-steady branch connects the first bifurcation to the second neighbouring bifurcation at $\mathrm{T}=\mathrm{T}_{1}=\mathrm{T}_{0}+2 \Upsilon$. For sufficiently small $\Upsilon$, the bifurcation at $\mathrm{T}=\mathrm{T}_{0}$ is supercritical. Allowing for a change of variables, Harris et al. (2000) eq. (3.8) shows that the amplitude of the solution is exponentially small with

$$
-\ln \left(|a|^{2}\right) \approx(2 \Upsilon)^{-3} \quad \text { for } \quad \Upsilon \ll 1
$$

and again irrelevant as $\Upsilon \downarrow 0$.

Harris et al. (2003) provided a comprehensive study of the subsequent bifurcation sequences that follow for various values of $\Upsilon$. Though an initial bifurcation to a simple SB Hocking and Skiepko (1981) solution was possible, for the small values of $\Upsilon$ of interest a super-critical SP-Hopf bifurcation occurs first, at lower $\mathrm{T}$, to two vacillating states with non-zero means.

The development of Harris et al. (2003) was largely numerical, but the results found for $\Upsilon=\frac{1}{4}$ are suggestive of the behaviours that might be expected for small $\Upsilon$. They show that with increasing $\mathrm{T}$ the frequency of the SP-vacillating modes decreases to zero forming two homoclinic orbits that glue and then a single orbit emerges subcritically. The ensuing SPsolutions are characterised by the expansion (33a) with $\Omega=0$ (see (34a,b)). Despite the fact that the emergent zero mean limit cycle is generally unstable, it gives the first real clue about the nature of the finite amplitude solutions that will occur as $\Upsilon \downarrow 0$.

The single frequency SP-limit cycle branch was followed to lower values of $\mathrm{T}$. It reached a minimum $\mathrm{T}$ and returned with a larger amplitude solution. Only small windows of stability were found. However, on solving (45) numerically as an initial value problem, Harris et al. 


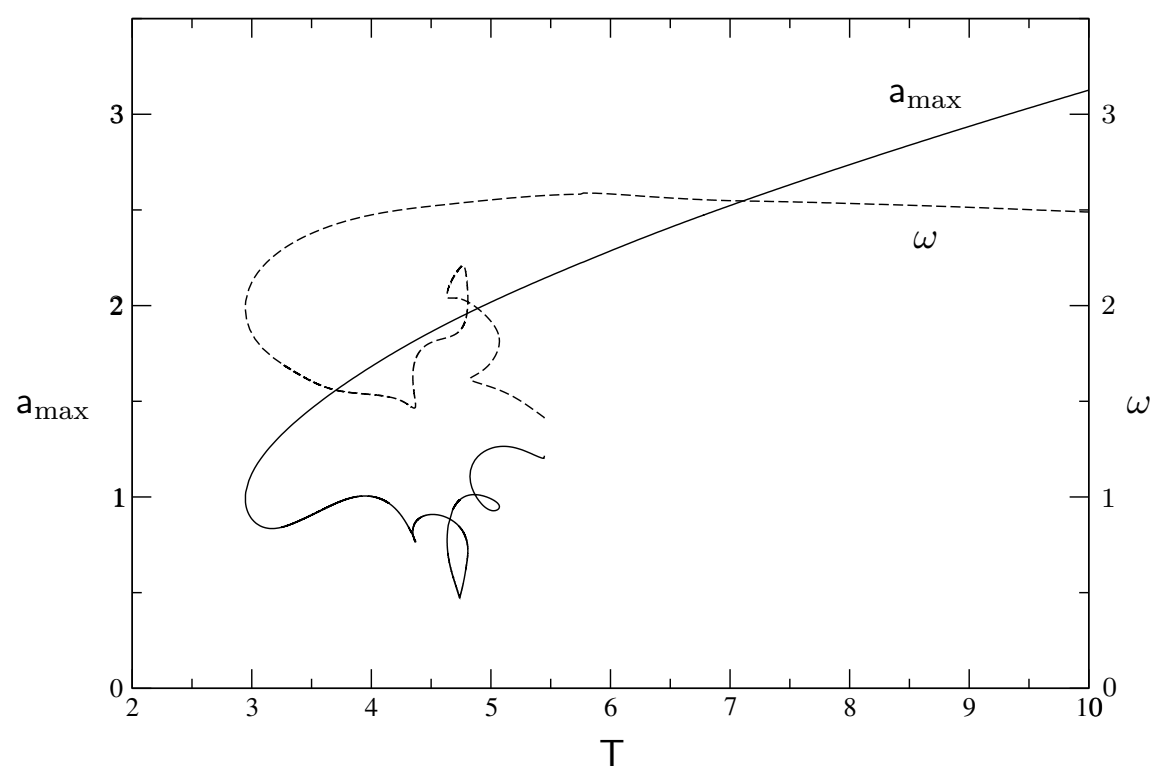

Figure 1. The maximum amplitude $\mathrm{a}_{\max }$ (solid) and frequency $\omega$ (broken) vs. T for the SP-solutions (51) for $\Upsilon=\frac{1}{4}$. (Reproduced from Blockley et al. 2007, fig. 1).

(2003) were generally only able to find SB-solutions of the type

$$
\mathrm{a}(\mathrm{x}, t)=\mathrm{e}^{\mathrm{i} \mathrm{x}_{0} t} \sum_{l=-\infty}^{\infty} \sum_{m=0}^{M-1} \mathrm{~b}_{l}^{m}(\mathrm{x}) \exp \left(\mathrm{i}\left(l+\frac{m}{M}\right) \omega t\right),
$$

with drifting phase angle $x_{0} t$ and characterised by a fundamental frequency $\omega$ describing the basic cycle but modified on a longer time scale characterised by a second frequency $\omega / M$ for some integer $M$. As a result the complete period $2 \pi M / \omega$ of $\mathrm{b}(x, t)=e^{-\mathrm{i} \times_{0} t} \mathrm{a}(\mathrm{x}, t)$ could be very long as exemplified by the large values of $M$ quoted by Harris et al. (2003). Their main findings are reproduced here for convenience (after appropriate scaling) in our table 2, where $a_{\max }$ denotes the maximum value of $|a(0, t)|$ over the entire period of oscillation. Although Harris et al. predominantly found SB-solutions they did identify a single SP-solution with $\Upsilon=\frac{1}{4}, \mathrm{~T}=3.975$.

An important suggestion made by Harris et al. (2003) is that of the possibility of the superposition of spatially overlapping modes with different frequencies. In other words, for a solution of the type (50) each mode $\mathrm{b}_{l}^{m}(\mathrm{x})$ is localised as a pulse in the vicinity of $\mathrm{x}=\mathrm{x}_{0}+l \omega$ for $-\infty<l<\infty$ on the length scale $\mathrm{O}(\omega)=\mathrm{O}(1)$. In fig. 21(d) of Harris et al. (2003) three pulses are clearly identifiable. The rôle of the small beating frequency $\omega / M$ is not so clear but may be simply manifest as a secondary unsteadiness of the pulse structure. It leads to the rough idea that, in the vicinity of any location $x=x_{0}+l \omega$, the dominant frequency is the same $x_{0}+l \omega$. In turn, that idea suggests the notion of a frequency staircase with the width and height of each step $\omega$. This is a notion that we will make more precise in what follows.

Subsequent calculations by Blockley et al. (2007) found a simple SP-solution of (45) of the type (50) with $\Upsilon=\frac{1}{4}, M=1$ and $\mathrm{x}_{0}=\omega / 2$. On setting $\mathrm{b}_{l}^{0}(\mathrm{x})=\mathrm{a}_{2 n}(\mathrm{x})$ (even $l$ ), $\mathrm{b}_{2 n-1}^{0}(\mathrm{x})=\mathrm{a}_{-2 n}^{*}(-\mathrm{x})($ odd $l) \forall n$, it takes on the form (33a) with $(34 \mathrm{a}, \mathrm{b})$ :

$$
\mathrm{a}(\mathrm{x}, t)=\sum_{n=-\infty}^{\infty} \mathrm{a}_{2 n}(\mathrm{x}) \exp \left(\mathrm{i}\left(2 n+\frac{1}{2}\right) \omega t\right)+\text { c.c.r. }
$$


where c.c.r. is the complex conjugate reflection

$$
\text { c.c.r. }=\sum_{n=-\infty}^{\infty} \mathrm{a}_{2 n}^{*}(-\mathrm{x}) \exp \left(-\mathrm{i}\left(2 n+\frac{1}{2}\right) \omega t\right) \text {. }
$$

This solution was followed by varying $\mathrm{T}$ and a plot of $\mathrm{a}_{\max }$ taken from Blockley et al. (2007) is reproduced here in figure 1. The crucial point is that the solution branch appears to be disconnected from any bifurcation sequence from the trivial zero-amplitude state.

\subsection{The generic case $\delta=\mathrm{O}(1)$}

The existence of SP-solutions like (51) of (45) for $\Upsilon=\frac{1}{4}$ provide clear evidence that such solutions continue to exist as $\Upsilon \downarrow 0$. Indeed, via the homotopy proposed in Section 3.5, the most important solutions of (1) appear to be of the form (51), following the change of variables $\mathrm{T} \mapsto \lambda, \mathrm{a} \mapsto a, \mathrm{a}_{2 n} \mapsto a_{2 n}$ and $\mathrm{x} \mapsto x$. In view of the important translational property (3) of the solutions of (1), the forms

$$
a_{2 n}(x)=\mathrm{e}^{\mathrm{i} \pi / 4} \bar{a}\left(x-x_{2 n}\right), \quad \text { where } \quad x_{l}=\left(l+\frac{1}{2}\right) \omega,
$$

are possible provided that $\bar{a}$ has the SP-property $\bar{a}^{*}(-x)=\bar{a}(x)$; at this stage, the factor $\mathrm{e}^{\mathrm{i} \pi / 4}$ is irrelevant being absorbable into the definition of $\bar{a}$. The constructed solution constitutes a homogeneous pulse train. In fact the solution (51) of (45) in Blockley et al. (2007) for finite $\Upsilon$ followed the discovery of the solutions with the special property (52) of (1) in Bassom and Soward (2004). Not surprisingly, the feature that the $\Upsilon=\frac{1}{4}$ solution branch illustrated in figure 1 appears to be disconnected to any bifurcation from the trivial zero-amplitude state, is certainly the case for the solutions of (1) which by necessity have no such bifurcation from the $a_{\max }=0$ state. These hanging branches are similar to those for pCf (see, e.g., fig. 19 in Waleffe 2003) and for Poiseuille flow (see, e.g., fig. 4 in Faisst and Eckhardt 2003).

In section 3.4, we made it clear that when $\delta=\mathrm{O}(1)$, there are three distinct length scales: $\ell_{c}=\mathrm{O}(\varepsilon), \ell_{p m}=\mathrm{O}\left(\varepsilon^{2 / 3}\right)$ and $\theta_{u s}=\mathrm{O}\left(\varepsilon^{1 / 3}\right)$ such that $\theta_{u s} \gg \ell_{p m} \gg \ell_{c}$ (recall table 1$)$. The pulse train solutions just described exist on the intermediate phase mixing length scale $\ell_{p m}$ relative to a moving origin.

Before continuing, we remark that other CGLe's, in which phase mixing is the only spatially inhomogeneous ingredient, have been investigated and also found to possess isolated pulse solutions (see Soward 1977, Ewen and Soward 1994a). So it has been perplexing to find that our narrow-gap sCf-CGLe does not allow them. Though it is natural to find solutions represented by a Fourier series in time, the fact that each frequency defines a pulse centred at different locations (our frequency staircase) is significant, because it means that the interaction of neighbouring pulses is necessary for their existence. Put another way, we cannot find widely separated pulse solutions; some overlap is necessary. The point that we wish to emphasise is that isolated pulses are the natural structures in narrow-gap sCf, just as streaks and rolls would be in $\mathrm{pCf}$ and neither system accepts their natural solutions. In $\mathrm{pCf}$ the streaks and rolls need to be supported by waves, whereas in sCf the pulses need to support each other. The ingredients of the mix differ in $\mathrm{pCf}$ (waves/streaks/rolls), whereas they are the same in $\mathrm{sCf}$ (all pulses). In both cases, the complete description is by some form of harmonic expansion partitioned to identify the ingredients; primarily by wave numbers for pCf (see (12)) and frequency $\omega=L$ for sCf. Of course the detailed comparisons noted are no more than curiosities, but are nonetheless intriguing. The key common issue at the root of it all is the unusual CGLe (1) for sCf which, like (8) governing the onset of instability for pCf, has no threshold value of $\lambda$ for instability, i.e. linear (infinitesimal) solutions lead to decay for all $\lambda$. 


\section{Pulse trains}

We now explore the properties of our pulse-trains more thoroughly and within this section we are only concerned with the solutions on the intermediate length scale $\ell_{p m}$ governed by (1) which applies after transforming to a moving origin (see (40a-d)). Two linearly independent infinitesimal amplitude steady solutions of (1) are

$$
a_{ \pm}(x)=\operatorname{Ai}\left( \pm e^{\mp \mathrm{i} \pi / 6}(x-\mathrm{i} \lambda)\right)
$$

Their asymptotic behaviours ${ }^{1}$ are such that each $a_{ \pm}(x) \rightarrow 0$ respectively as $x \rightarrow \pm \infty:$

$$
a_{ \pm} \propto \exp \left(-\frac{2}{3} e^{\mp \mathrm{i} \pi / 4}(x-\mathrm{i} \lambda)^{3 / 2}\right)
$$

up to multiplication by an algebraic function but with each diverging as $x \uparrow \infty$ or as $x \downarrow-\infty$ for all finite $\lambda$ real or complex. So there is no linear combination of $a_{ \pm}(x)$ that remains bounded for all $x$, i.e., there is no acceptable linear solution. Nevertheless, as mentioned at the end of the previous section 4.2, there have been precedents for individual pulse solutions for other CGLe's with phase mixing. So it was a disappointment when Hocking (private communication, circa 1980) sought such localised steady pulse solutions of the fully nonlinear version of (1) that converged to zero like $a_{ \pm}(x)$ as $x \rightarrow \pm \infty$, and found all his efforts to no avail. That and all subsequent attempts strongly indicate that no such solutions exist. Nevertheless, once time-dependent solutions are admitted the notion of pulses in the form of pulse trains becomes a realistic possibility.

In our discussion of the nature of the solutions of the full and reduced CGLe's (25a) and (45) we laid considerable emphasis to the rôle of SP-modes (see (30)) largely due to their relative simplicity. However the results of Harris et al. (2003) at small $\Upsilon$ suggest that SB finite amplitude numerical solutions of (45) seem to be the rule rather than the exception. The reason is obvious: for in the limit $\Upsilon \downarrow 0$ to which (1) applies, new solutions $\mathrm{e}^{\mathrm{i} \Omega t} a(x-\Omega, t)$ generated by the translational property (3) from an SP-solution $a(x, t)$ will themselves be SB for all constant $\Omega$. With that caveat in mind, our objective here is to identify periodic SP-solutions which should be regarded as generators of the wider sub-class of SB-solutions of the form (3).

\subsection{The continuous frequency $x$}

A natural starting point for understanding the nature of solutions to (1) is to consider modulations of the leading-order phase mixing solution $\mathrm{e}^{\mathrm{i} x t}$. At least it is the leading-order solution for large $|x|$ and certainly on the even longer length scale $\mathrm{O}\left(\Upsilon^{-1}\right)$ of the true unstable range. To that end we note that

$$
a(x, t)=\mathrm{e}^{\mathrm{i} x t} \mathcal{A}(x, t)
$$

(cf. Ewen and Soward 1994b, eq. (1.12a)) is a solution provided that $\mathcal{A}(x, t)$ solves

$$
\frac{\partial \mathcal{A}}{\partial t}=\lambda \mathcal{A}+\mathfrak{D}^{2} \mathcal{A}-|\mathcal{A}|^{2} \mathcal{A}, \quad \mathfrak{D} \equiv \frac{\partial}{\partial x}+\mathrm{i} t
$$

and, like $a(x, t)$, remains bounded as $|x| \rightarrow \infty$. So, if the initial condition is a harmonic wave $a(x, 0)=\mathcal{A}(x, 0)=\mathrm{e}^{\mathrm{i} k x}$ with constant wave number $k$, then $\mathcal{A}$ evolves to

$$
\mathcal{A}(x, t)=A(k, t) \mathrm{e}^{\mathrm{i} k x}
$$

\footnotetext{
${ }^{1}$ Note that the square root in $(53 \mathrm{~b})$ is taken such that $(x-\mathrm{i} \lambda)^{3 / 2} /|x|^{3 / 2} \rightarrow 1$ as $x \rightarrow \pm \infty$.
} 
where $A(k, t)$ solves

$$
\frac{\partial A}{\partial t}=\left(\lambda-K^{2}-|A|^{2}\right) A \quad \text { with } \quad K(t)=t+k .
$$

The general solution $A(k, t)$ is given by eq. (3.16) of Bassom and Soward (2004), but its properties, particularly its necessary collapse as $t \uparrow \infty$, are readily appreciated. The nonlinear coefficient $-|A|^{2}$ of $A$ leads to amplitude quenching in the usual way. However, even in the absence of this quenching term, infinitesimal (i.e. linear) solutions

$$
A \propto \exp \left[\lambda K-\frac{1}{3} K^{3}\right]
$$

are forced to decay at the ever-increasing rate $-K^{2}=-(t+k)^{2}$ once $t>-k$. Thus the extraction of the factor $\mathrm{e}^{\mathrm{i} x t}$ in (54) emphasises the rôle of phase mixing, which causes the entire solution (54) to possess the time-dependent wave number $K$ and wavelength $2 \pi / K$, which after $t=-k$ decreases indefinitely leading to enhanced dissipation. Indeed, given the arbitrary bounded initial condition $a(x, 0)=\int_{-\infty}^{\infty} A(k, 0) \mathrm{e}^{\mathrm{i} k x} \mathrm{~d} x$ the linear solution is

$$
a(x, t)=\int_{-\infty}^{\infty} A(k, 0) \exp \left[\lambda t-\frac{1}{3}\left[(t+k)^{3}-k^{3}\right]\right] \mathrm{e}^{\mathrm{i}(t+k) x} \mathrm{~d} k,
$$

which necessarily decays, e.g., the fundamental solution for $A(k, 0)=e^{-\mathrm{i} k L} /(2 \pi)$ :

$$
a(x, t ; L)=\frac{\exp \left(-t^{3} / 12+\lambda t\right)}{\sqrt{4 \pi t}} \exp \left(-\frac{(x-L)^{2}}{4 t}+\mathrm{i} \frac{t(x+L)}{2}\right),
$$

with initial value $a(x, 0 ; L)=\delta(x-L)$, where $\delta(x)$ is the Dirac Delta function and $L$ is an arbitrary constant. The notion of a time-dependent local wave number $K(t)=t+k$ is a well known concept with origins in the work of Lord Kelvin (1887) on sheared plane waves. It has repercussions in a variety of contexts where shear or phase mixing cause the local wave number $K(t)$ to increase with time, for example in geophysical fluid flows (e.g., Farrell and Ioannou 1993) and astrophysics (e.g., Leprovost and Kim 2009, Dubrulle and Knobloch 1992, Heinemann et al. 2011).

\section{2. $\quad$ Fourier series in $x$ of wavelength $2 L=2 \pi / T$}

Next, instead of the single mode $\mathcal{A}(x, t)=A(k, t) \mathrm{e}^{\mathrm{i} k x}$, we consider the Fourier series

$$
\mathcal{A}(x, t)=\sum_{n=-\infty}^{\infty} A_{n}(t) \mathrm{e}^{\mathrm{i} n T x}, \quad \operatorname{Im}\left\{A_{n}(t)\right\}=0,
$$

(cf. Ewen and Soward 1994b, eq. (1.13a)), with constant wave-number $T$ and wavelength $2 L=2 \pi / T$. By requiring each $A_{n}(t)$ to be real, we ensure that the corresponding $a(x, t)$ is an SP-mode. On substitution of (61a) into (55) and noting (61b), we obtain

$$
\frac{\mathrm{d} A_{n}}{\mathrm{~d} t}=\left(\lambda-(t+n T)^{2}\right) A_{n}-\sum_{\alpha=-\infty}^{\infty} \sum_{\beta=-\infty}^{\infty} A_{n+\alpha} A_{n+\alpha+\beta} A_{n+\beta} .
$$

In view of the failure of the single mode (56) to persist, the hope is that the sum (61a) can survive via the nonlinear interaction with neighbouring modes. The essential idea is that each $A_{n}(t)$ is localised temporally about $t=-n T$ and tends to zero as $t+n T \rightarrow \pm \infty$. So, on the interval $-(n+1) T \leq t \leq-n T$, we expect only a limited band of modes $A_{-\alpha-n}(t)$, with $-\bar{\alpha} \leq \alpha \leq \bar{\alpha}$ for some sufficiently large $\bar{\alpha}$, not to have exponentially small amplitude. Hence, for the numerical integration of (62), we only keep those modes on each time interval $-(n+1) T \leq t \leq-n T$ and the rest we set to zero. At $t=-n T$, we drop the mode $A_{\bar{\alpha}-n}(t)$ and 
include the new mode $A_{-(\bar{\alpha}+1)-n}(t)$ with initial value $A_{-\bar{\alpha}-n-1}(-n T)=0$. Then on the next interval $-n T \leq t \leq(-n+1) T$, we solve for the modes $A_{-\alpha-(n+1)}(t)$ again with $-\bar{\alpha} \leq \alpha \leq \bar{\alpha}$. On each successive time interval $T$, we repeat the process of dropping a mode and introducing a new one. This procedure is similar to the Shearing Box method used frequently in astrophysics, particularly in the study of accretion discs (see, e.g., Hawley et al. 1995, and fig. 1 therein), which is often modelled by a Keplerian shearing sheet (see, e.g., fig. 1 of Riols et al. 2013, together with the numerous references therein).

An inspection of $\mathrm{e}^{\mathrm{i} x t} \mathcal{A}(x, t)$ shows that $\mathcal{A}(x, t)$ satisfies

$$
\mathcal{A}(x, t+N T)=\sum_{n=-\infty}^{\infty} A_{n-N}(t+N T) \mathrm{e}^{\mathrm{i} n T x},
$$

for any integer $N$. A solution is periodic, if $A_{n-N}(t+N T)=A_{n}(t)$, for some integer $N$ and for all $A_{n}$ and all time $t$. The smallest value of $N(>0)$, for which this occurs defines the period $N T$ of the solution.

In contrast to the Ewen and Soward (1994b) study of a related but distinct CGLe, which was restricted to $N=1$, the simplest solution found by Bassom and Soward had $N=4$ and was generated by a single function $\widehat{A}(t)$ (see Bassom and Soward 2004, eqs. (3.7), (3.8)):

$$
A_{n}(t)=\mu_{n} \widehat{A}(t+n T), \quad \text { where } \quad \mu_{n}=(-1)^{n(n-1) / 2},
$$

for all integer $n$; note that $\mu_{n+1}=(-1)^{n} \mu_{n}$ with $\mu_{0}=1$. The function $\widehat{A}(t)$ itself satisfies

$$
\frac{\mathrm{d} \widehat{A}}{\mathrm{~d} t}=\left(\lambda-t^{2}\right) \widehat{A}-\sum_{\alpha=-\infty}^{\infty} \sum_{\beta=-\infty}^{\infty}(-1)^{\alpha \beta} \widehat{A}(t+\alpha T) \widehat{A}(t+(\alpha+\beta) T) \widehat{A}(t+\beta T),
$$

which for particular values of $\lambda$ and $T$ has non-zero solutions satisfying $\widehat{A} \rightarrow 0$ as $t \rightarrow \pm \infty$. The resulting form for (54) using (61) is

$$
a(x, t)=\exp (t x) \sum_{n=-\infty}^{\infty} \mu_{n} \widehat{A}(t+n T) \exp (\mathrm{i} n T x) .
$$

With the wavelength defined to be $2 L(=2 \pi / T)$, we have

$$
T L=\pi \quad \Longrightarrow \quad \mathrm{e}^{\mathrm{i} n T L}=(-1)^{n} \text {. }
$$

Used together with the relations $(64 a, b)$, it is readily shown that the solution (66) possesses the fundamental symmetries

$$
a(x+L, t \pm T)=\mp \mathrm{e}^{\mathrm{i} L t} a(x, t),
$$

whose repeated use determines

$$
a(x+2 L, t)=e^{2 \mathrm{i} L t} a(x, t), \quad a(x, t+2 T)=-a(x, t) .
$$

The former (68b) confirms the spatial periodicity of $\mathcal{A}(x, t)=e^{-\mathrm{i} x t} a(x, t)$ over the wavelength $2 L$, while the latter $(68 \mathrm{c})$ shows that $a(x, t)$ has zero temporal mean with half (full) temporal period $2 T(4 T)$.

As a measure of the solution amplitude we introduce the space average of the energy $|a(x, t)|^{2}$ which, upon integration of the series representation (66) over the periodicity length $2 L=$ $2 \pi / T$, is

$$
\widehat{\mathcal{E}}(t)=\frac{1}{2 L} \int_{-L}^{L}|a(x, t)|^{2} \mathrm{~d} x=\sum_{n=-\infty}^{\infty}|\widehat{A}(t+n T)|^{2} .
$$

Its temporal period $T$ reflects the periodicity of the modulus of (68a), $|a(x+L, t+T)|=|a(x, t)|$ for which the $x$-origin shift is irrelevant after integration with respect to $x$. From (69a) we 


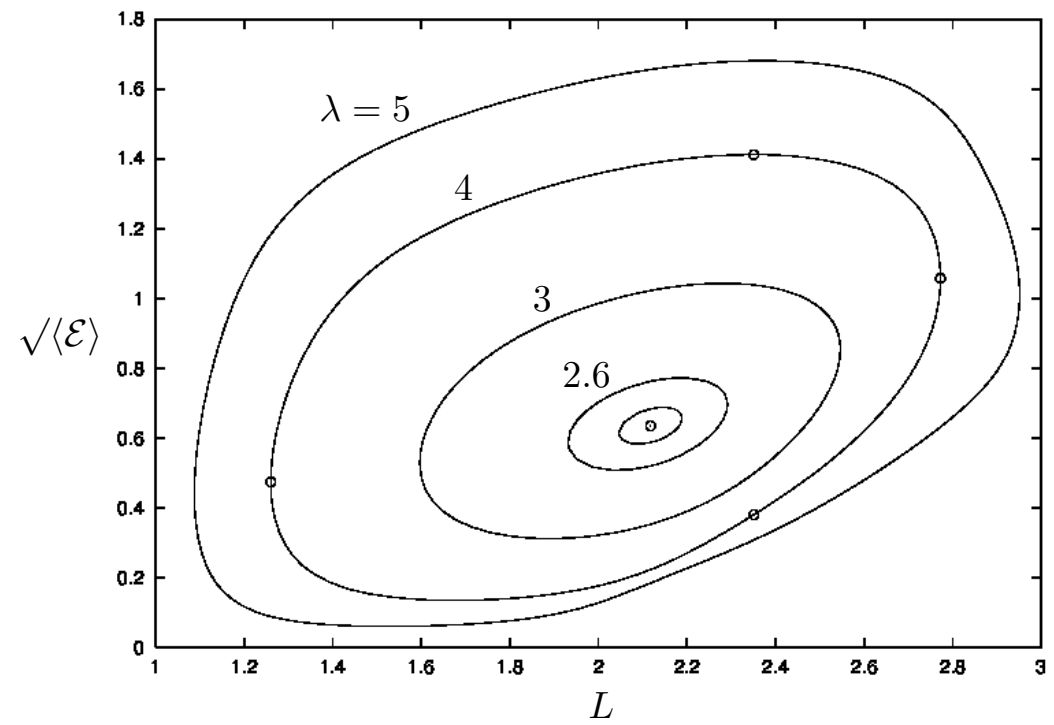

Figure 2. Plots of $\sqrt{ }\langle\mathcal{E}\rangle$ vs. $L$ for various values of $\lambda=2.55$ (innermost curve), 2.6, 3, 4, and 5 (outermost). The central o identifies the $A_{n}(t)$-solutions for $\left.\lambda=2.541 \doteqdot \lambda_{\text {inf }}\right)$ plotted on figure 3 . The other four o's on the curve for $\lambda=4$ identify other $A_{n}(t)$-solution plots illustrated in figures 4, 6, 7, 8 of Bassom and Soward (2004). (Reproduced from Bassom and Soward 2004, fig. 1).

can define the space-time average

$$
\langle\mathcal{E}\rangle=\frac{1}{T} \int_{0}^{T} \widehat{\mathcal{E}}(t) \mathrm{d} t=\frac{1}{T} \int_{-\infty}^{\infty}|\widehat{A}(t)|^{2} \mathrm{~d} t .
$$

On increasing $\lambda$, Bassom and Soward (2004) showed that the solutions (66) first occur at $\lambda=\lambda_{\text {inf }}$ :

$$
\lambda_{\mathrm{inf}} \approx 2.5407,
$$

for which the corresponding values of $L$ and $\sqrt{ }\langle\mathcal{E}\rangle$ are $L_{\text {inf }} \approx 2.1183$ and $\sqrt{ }\langle\mathcal{E}\rangle_{\text {inf }} \approx 0.6344$. For $\lambda>\lambda_{\text {inf }}$ solutions were found on a range of $L$-values, and on that range there were two solutions for each $L$. We plot $\sqrt{ }\langle\mathcal{E}\rangle$ versus $L$ for various values of $\lambda$ on figure 2 .

The sign reversal of $-A_{n}(t)=-\mu_{n} \widehat{A}(t+n t)$ as $n$ increases by 2 , visible for the case $\lambda=2.541 \doteqdot \lambda_{\text {inf }}$ on figure 3 , appears to be a key feature of the solution. The excitation of small correctly-signed $\widehat{A}(t)$, when $t<-\sqrt{\lambda}(\approx-1.594$ for figure 3$)$ where the linear growth rate $\lambda-t^{2}$ in (65) is negative, needs a source of the same sign. It is provided by the nonlinear contribution

$$
\widehat{A}^{2}(t+T) \widehat{A}(t+2 T),
$$

$(T \approx 1.483)$ to the infinite double sum on the right-hand side of $(65)$, namely the $\alpha=\beta=1$ term for which $(-1)^{\alpha \beta}=-1$.

\subsection{Pulse train solutions: Fourier series in $t$, half period $2 T=2 \pi / L$}

Though the numerical determination of the SP-solution (66), i.e., $a(x, t)=\mathrm{e}^{\mathrm{i} x t} \mathcal{A}(x, t)$ with $\mathcal{A}(x, t)=\sum_{n=-\infty}^{\infty} \mu_{n} \widehat{A}(t+n T) \mathrm{e}^{\mathrm{i} n T x}$ was straightforward, its physical interpretation is rather opaque. In Appendices A and B of Bassom and Soward (2004) it was shown that our Fourier series (66) in $x$ allows for an alternative (and perhaps more illuminating) representation as a Fourier series in $t$. Though the derivation in those Appendices is direct, it is somewhat convoluted and conveys the impression that the result is fortuitous. Here, in our Appendix, we 


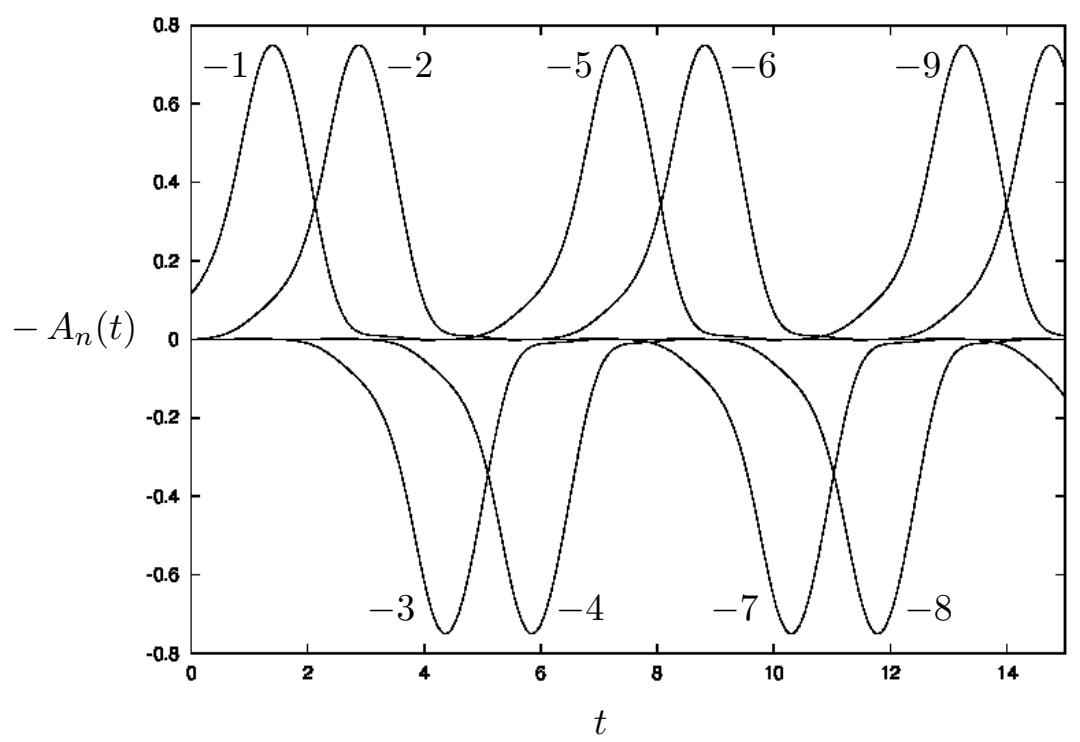

Figure 3. The $\lambda=2.541, L=2.118, \sqrt{ }\langle\mathcal{E}\rangle=0.6344$ solution with $T=1.4833$ identified by the central $\circ$ on figure 2 . The time series of the amplitudes $-A_{n}(t)=-\mu_{n} \widehat{A}(t+n T)$ for $n=-1,-2, \cdots-9$, as labelled. (Reproduced from Bassom and Soward 2004, fig. 2a).

provide an alternative derivation using Fourier transform methods which adds much insight. Bizarrely all the even $n$ terms in the expansion (66) cancel each other out (see (A.6)) and only the odd $n$ terms survive, leading to the alternative representation

$$
a(x, t)=\sum_{n=-\infty}^{\infty} \bar{a}\left(x-x_{n}\right) \exp \left[\mathrm{i}\left(x_{n} t+(-1)^{n} \frac{1}{4} \pi\right)\right], \quad x_{n}=\left(n+\frac{1}{2}\right) L
$$

(see (A.9)), where the Fourier transform of $\bar{a}(x)$ is

$$
\widehat{A}(t)=\mathcal{F}\{\bar{a}\}(t)=\frac{1}{\sqrt{2} L} \int_{-\infty}^{\infty} \bar{a}(x) e^{-\mathrm{i} x t} \mathrm{~d} x
$$

and $\bar{a}(x)$ itself is the inverse Fourier transform of $\widehat{A}(t)$ :

$$
\bar{a}(x)=\mathcal{F}^{-1}\{\widehat{A}\}(x)=\frac{1}{\sqrt{2} T} \int_{-\infty}^{\infty} \widehat{A}(t) \mathrm{e}^{\mathrm{i} x t} \mathrm{~d} t \quad(L T=\pi)
$$

(see $\bar{a}(x)$ illustrated in figure 4 for the $\widehat{A}(t)$ used in figure 3$)$. Since $\widehat{A}(t)$ is real (see $(61 \mathrm{~b})$ ), (73b) implies that $\bar{a}(x)$ has the SP-property $\bar{a}(x)=\bar{a}^{*}(-x)$. Significantly the period of oscillation of the mode with frequency $x_{n}$ in (72) is

$$
t_{n}=\frac{2 \pi}{\left|x_{n}\right|}=\frac{4 T}{|2 n+1|} .
$$

Indeed these periods are readily identified in figure 5 on the vertical lines $x / L= \pm \frac{1}{2}, \pm \frac{3}{2}$ and $\pm \frac{5}{2}$ along which $t_{n} / T=4, \frac{4}{3}$ and $\frac{4}{5}$ respectively. Of course, $t_{0}=4 T$ is the complete temporal period of the pulse train manifest on the axis $x=0$.

Noting that $x_{2 n-1}=-x_{-2 n}$ and $\bar{a}\left(x-x_{2 n-1}\right)=\bar{a}\left(x+x_{-2 n}\right)$, we may express (72) in the alternative form

$$
a(x, t)=\mathrm{e}^{\mathrm{i} \pi / 4} \sum_{n=-\infty}^{\infty} \bar{a}\left(x-x_{2 n}\right) \exp \left(\mathrm{i} x_{2 n} t\right)+\text { c.c.r. },
$$




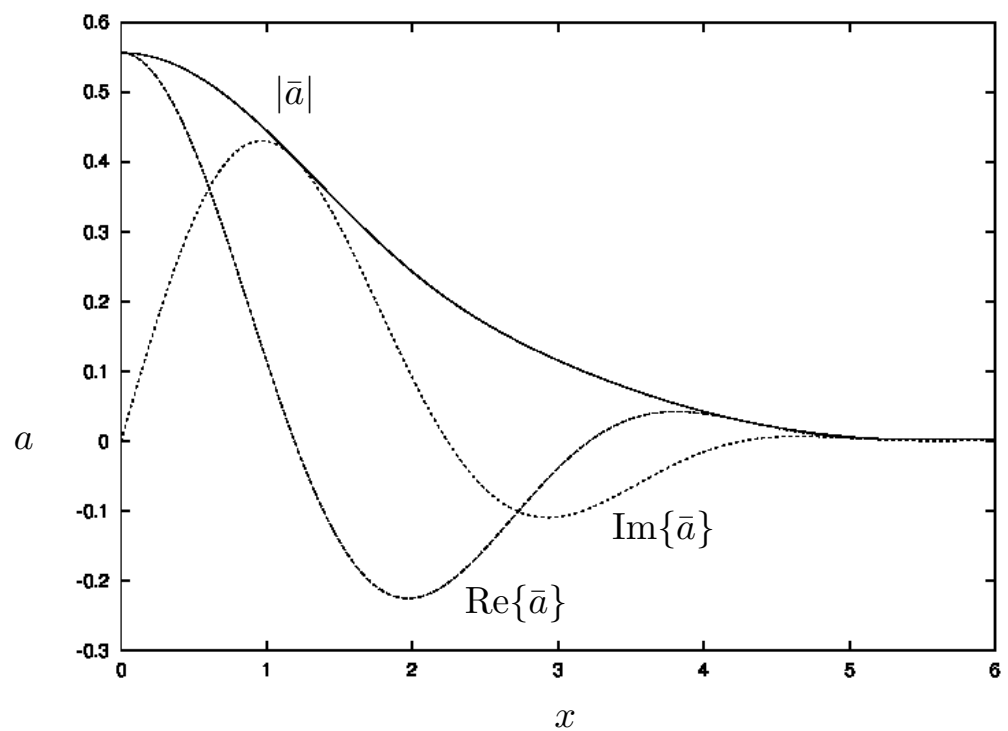

Figure 4. The pulse $\bar{a}(x)$ defined by (73b) for $\widehat{A}(t)$ employed in figure 3 with $L=2.118$. The modulus $|\bar{a}(x)|$, real part $\operatorname{Re}\{\bar{a}(x)\}$ and imaginary part $\operatorname{Im}\{\bar{a}(x)\}$ are identified by the continuous and long and short dashed lines respectively. (Reproduced from Bassom and Soward 2004, fig. 2b).

where

$$
\text { c.c.r. }=e^{-\mathrm{i} \pi / 4} \sum_{n=-\infty}^{\infty} \bar{a}\left(x+x_{2 n}\right) \exp \left(-\mathrm{i} x_{2 n} t\right)
$$

in which we have replaced $-2 n$ by $2 n$; remember that $\bar{a}^{*}(-x)=\bar{a}(x)$. The elegant form of $(75 \mathrm{a}, \mathrm{b})$ coincides with $(51 \mathrm{a}, \mathrm{b})$ when $a_{2 n}(x)=\mathrm{e}^{\mathrm{i} \pi / 4} \bar{a}\left(x-x_{2 n}\right)$ (see (52)) and $\omega=L$.

Taking $a(x, t)$ in its primitive form $(72)$, it is readily established that the time average of $|a(x, t)|^{2}$ over the half-period $2 T=2 \pi / L$ is

$$
\overline{\mathcal{E}}(x) \equiv \frac{1}{2 T} \int_{-T}^{T}|a(x, t)|^{2} \mathrm{~d} t=\sum_{n=-\infty}^{\infty}\left|\bar{a}\left(x-x_{n}\right)\right|^{2},
$$

which is spatially periodic over the distance $x_{n+1}-x_{n}=L$. So integration with respect to $x$ from 0 to $L$ determines the space-time average

$$
\langle\mathcal{E}\rangle=\frac{1}{L} \int_{0}^{L} \overline{\mathcal{E}}(x) \mathrm{d} x=\frac{1}{L} \int_{-\infty}^{\infty}|\bar{a}(x)|^{2} \mathrm{~d} x .
$$

In view of Parseval's theorem for Fourier transforms, this result agrees with (69b).

Finally we remark that direct substitution of (75) into the CGLe (1) shows that $\bar{a}(x)$ satisfies

$$
\left(\frac{\mathrm{d}^{2}}{\mathrm{~d} x^{2}}+\lambda+\mathrm{i} x\right) \bar{a}(x)=\sum_{m=-\infty}^{\infty} \sum_{n=-\infty}^{\infty}(-1)^{m n} \bar{a}(x-m L) \bar{a}^{*}(x-(m+n) L) \bar{a}(x-n L)
$$

(see Bassom and Soward 2004, eqs. (2.25a,b)). Inspection of figure 4 with right-hand axis $x=6<3 L$, albeit for a very low $\lambda=2.541$ close to $\lambda_{\text {inf }}$ (see $(70)$ ), suggests that only the pulse interactions of close neighbours are important. Under the most severe truncation of the double sum in (78) that only retains the $m=n=0$ term $|\bar{a}(x)|^{2} \bar{a}(x)$, the equation reduces to the time-independent version of (1) which, as we commented there, appears to have no pulse solution. So we need to look further at the pulses with centres displaced a distance $L$. All cases $m(n)=0, n(m)= \pm 1$, which each produce the same signed $2|\bar{a}(x \mp L)|^{2} \bar{a}(x)$ 

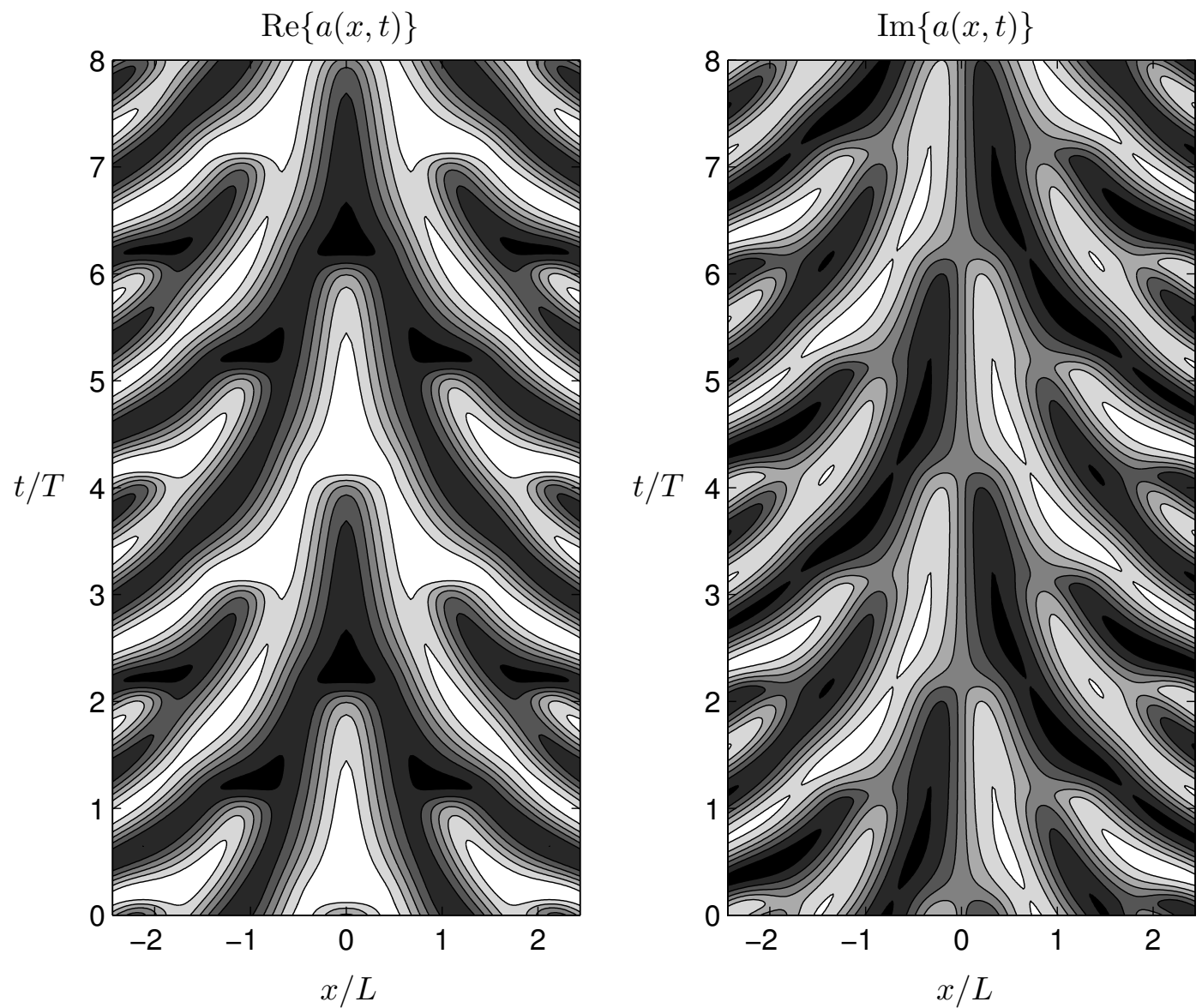

Figure 5. Contours of constant $\operatorname{Re}\{a(x, t)\}$ (left) and $\operatorname{Im}\{a(x, t)\}$ (right) in the $x / L-t / T$ plane for the $\lambda=10, T \approx 1.263$ case characterised by $L \approx 2.487, \sqrt{ }\langle\mathcal{E}\rangle \approx 2.3$ (see the coordinates of the solid dot on fig. 5 of Blockley et al. 2007 ). The time origin has been shifted, $t \mapsto t-q T$ (for some suitable non-integer $q$ ), to ensure that $\operatorname{Re}\{a\}(0,0)=0$. (Reproduced from Blockley et al. 2007, fig. 3(b)).

like $|\bar{a}(x)|^{2} \bar{a}(x)$, are probably unhelpful, but see below. By similar reasoning, of those pulses with maximum separation $2 L$, the most likely candidate responsible for the existence of the solution is the coupling of the $|m|=1,|n|=1$ pulses (not $m(n)=0, n(m)= \pm 2$ ), which leads to the contribution

$$
-2 \bar{a}(x-L) \bar{a}^{*}(x) \bar{a}(x+L)-\bar{a}^{2}(x-L) \bar{a}^{*}(x-2 L)-\bar{a}^{2}(x+L) \bar{a}^{*}(x+2 L),
$$

to the right-hand side of (78).

In support of the proposals just made, we note that the simplest possible pulse train structure is associated with the $N=1$ case

$$
\mathcal{A}(x, t)=\sum_{n=-\infty}^{\infty} \widehat{A}(t+n T) \exp (\mathrm{i} n T x)
$$

adopted by Ewen and Soward (1994b) eqs. (1.13a), (1.15), equivalently $\mu_{n}=1$ in (64a) for all integer $n$. If it existed, $\widehat{A}(t)$ would be governed by (65) but with the coefficients $(-1)^{\alpha \beta}$ in the double sum all set to unity. Since we found no such solutions, the absence of the coefficient -1 for $\alpha=\beta=1$, leading to the nonlinear term $\widehat{A}^{2}(t+T) \widehat{A}(t+2 T)$ identified in (71) for our $N=4$ solutions, appears to be fatal.

To explore the argument further, we note that the Fourier series (80) leads to the pulse 
train structure

$$
a(x, t)=\sum_{n=-\infty}^{\infty} \bar{a}(x-n L) \exp (\text { in } L t) \quad(\text { Note: } \quad L T=2 \pi)
$$

(Ewen and Soward 1994b, eq. (1.5)). The amplitude function $\bar{a}(x)$ would then satisfy the amplitude equation (78) but with the factor $(-1)^{m n}$ omitted from the nonlinear term. The apparent absence of solutions of the type (81) suggests that the existence of our $N=4$ pulse train solution (72) depends upon the nonlinear coupling in (78) from the terms with $(-1)^{m n}=-1$ identified in (79).

\section{Discussion}

The essential point that figure 2 illustrates is that on increasing $\lambda$ there is a minimum value $\lambda_{\text {inf }}$ at which a finite amplitude solution with fundamental frequency $\omega=L_{\text {inf }}$ exists. For larger $\lambda\left(>\lambda_{\text {inf }}\right)$ such solutions exist over a finite range $L_{\min }(\lambda) \leq L \leq L_{\max }(\lambda)$ of fundamental frequencies $\omega=L$. Since for any realised configuration the value of $\lambda$ is a local property of the location of the origin $\mathrm{x}=\Upsilon^{-1} \mathrm{X}(t)$ (see (40a)), it effectively decreases in value from its maximum value $\lambda=\mathrm{T}$ at the equator to zero at $\mathrm{x}=\mathrm{x}_{\text {us }}$ at the edge of the unstable range. Evidently for the finite amplitude motion to exist, at the very least we need $\mathrm{T} \geq \lambda_{\text {inf }}$. So in view of (44) we expect the finite-amplitude disturbances to exist on the range given roughly by

$$
|\mathrm{x}| \lesssim \mathrm{x}_{\text {rge }}=\Upsilon^{-1} \mathrm{x}_{\text {rge }}, \quad \text { where } \quad \mathrm{x}_{\text {rge }}=\sqrt{\mathrm{T}-\lambda_{\text {inf }}},
$$

over which acceptable values of the frequency (also periodicity length) $L$ may vary slowly. With this interpretation of results, we may identify the minimum value of $\mathrm{T}$ with $\mathrm{T}_{\text {inf }}=\lambda_{\text {inf }}$ corresponding to which there is a minimum value of $\mathrm{Ta}=\mathrm{Ta} \mathrm{a}_{\text {inf }}$ for the existence of a finite amplitude solution with fundamental frequency $\omega=L_{\text {inf }}$ localised close to the equator.

In the case of pCf, from the few particular $\alpha, \gamma$ (see (12)) choices made in figure 19 in Waleffe (2003), it is clear that hyperplane $\alpha-\gamma$-amplitude (wall shear stress) plots for various values of Re based on more extensive data would yield surfaces similar (albeit in one extra dimension) to the narrow-gap sCf-curves plotted in our figure 2 for various values of $\lambda$.

Though only the limited set of solutions (75a,b) obtained by Bassom and Soward (2004) were reported in section 5, Blockley et al. (2007) investigated bifurcations from them and found other solutions that could be categorised within the class of solutions described by (50). Indeed, in view of the solutions of Harris et al. (2003) of initial value problems based on the small- $\Upsilon$ equation (45), we may expect a multitude of almost periodic solutions to exist with pulse like structures of width $L=\mathrm{O}(1)$, a view that gains support from the results of Blockley et al. (2007). Their existence by no mean guarantees the persistence of any of them over longer length scales and consequently longer time scales. Specifically, the solutions obtained in section 5 do not take account of variations over the longer length scale $\mathrm{O}\left(\Upsilon^{-1}\right)=\mathrm{O}\left(\delta^{2 / 3} \varepsilon^{-1 / 3}\right)($ see $(36 \mathrm{~b})$ and table 1$)$. Both the spatially varying $\Lambda\left(\mathrm{X}_{0}, 0\right)($ see $(44))$ and the spatially varying group velocity $-\mathcal{D} \Upsilon_{\times}$(see $(25 \mathrm{a})$ ) may disrupt any nice periodic solution found in Bassom and Soward (2004) and Blockley et al. (2007) on the long time and space scales. For example the spatially varying group velocity may cause the local $L$ to shorten and move outside the range that admits solutions. In so doing it may well be replaced by solutions with admissible $L$. A likely evolutionary scenario is that patches of pulses evolve possibly separated by dislocations, similar to that found in Bénard convection in layers of very large horizontal extent. The upshot is that the solutions are expected to be mildly chaotic but with a tendency to be attracted to the basic structures that we have described.

A nagging concern may remain that some of the non-uniformities mentioned could have 
been avoided by adopting a fixed local origin $\mathrm{x}_{0}=\Upsilon^{-1} \mathrm{X}_{0}$ (see (38b)) and investigating solutions of (39) instead. We can allay these worries by the following two comments. Firstly, the Taylor vortices are driven most vigorously when $\mathbf{X}_{0}$ is small. In that case (39) is adequately approximated by (1). Secondly, when $X_{0}$ is of order unity, some differences of the nature of the solutions will be apparent, but there is no reason to suppose that the solutions of (39) are any more relevant than those of (1).

Even the early studies of Harris et al. (2003) (see their fig. 21) clearly pointed to the existence of solutions dominated by pulses with distinct frequencies that increased with distance from the equator $\theta=0$. Indeed Bassom and Soward (2004) were able to quantify this by their solution (75) for which the frequency of the dominant mode at $\mathrm{x}$ is given formally by

$$
\omega(\mathrm{x})=L \operatorname{sgn}(\mathrm{x})\left(\frac{1}{2}+\sum_{n=1}^{\infty} \mathrm{H}(|\mathrm{x}|-n L)\right),
$$

where $\mathrm{H}(\mathrm{x})$ is the Heaviside Unit function, i.e., $\omega(-\mathrm{x})=-\omega(\mathrm{x})$ with $\omega(\mathrm{x})=\left(\frac{1}{2}+n\right) L$ on $n L<\mathrm{x}<(n+1) L$ for $n=0,1,2,, \cdots$. Since the Taylor vortices exist on the short

$$
\ell_{c} / \ell_{p m}=\mathrm{O}\left(\delta^{1 / 3} \varepsilon^{1 / 3}\right),
$$

$x$-length scale (see (35) and table 1), this means that the dominant frequency in a block (pulse) of Taylor vortices is synchronised to (83), a frequency staircase, rather than each Taylor vortex exhibiting a frequency increment that is tied to the continuous frequency $\omega(x)=x$. Indeed, the realised state in the small- $\varepsilon$ limit will be mildly chaotic but attracted to a staircase frequency somewhat similar to (83) albeit $L$ may possibly be a slowly varying function of $\mathrm{x}$ and $t$, as will the space origin. This discretisation of the frequency runs contrary to the often expressed point of view that, in unbounded systems with linear phase mixing, a frequency proportional to the coordinate is a useful ansatz for linearised disturbances (see, e.g., Leprovost and Kim 2009, Dubrulle and Knobloch 1992, Heinemann et al. 2011). Our study of (1) shows clearly that nonlinear systems prefer a frequency staircase instead.

The implications of the frequency staircase deserve elaboration. Specifically, consider the pulse contribution

$$
\bar{a}\left(\mathrm{x}-\mathrm{x}_{n}\right) \exp \left\{\mathrm{i}\left[\left(\ell_{p m} / \varepsilon\right) \breve{k}_{c} \mathrm{x}+\int \mathrm{x}_{n}(t) \mathrm{d} t+(-1)^{n} \frac{1}{4} \pi\right]\right\}
$$

from each individual mode $(n=\cdots,-2,-1,0,1,2, \cdots)$ in $(75)$, and where

$$
\mathrm{x}_{n}(t)=\Upsilon^{-1} \mathrm{X}(t)+x_{n} \quad \text { and } \quad x_{n}=\left(n+\frac{1}{2}\right) L
$$

(in a rather loose sense as $L$ might be a slowly varying function of position and time), to the primitive Taylor vortex form $a(\theta, \tau) \exp \left(\mathrm{i} \varepsilon^{-1} \breve{k}_{c} \theta\right)$ introduced in (19) but expressed in our $(\mathrm{x}, t)$ coordinates defined by $(24 \mathrm{a}, \mathrm{c})$. When $\bar{a}$ is written in amplitude and phase form, only the amplitude $|\bar{a}|$ is significant, as the $\mathrm{x}$-derivative of the argument $\arg \{\bar{a}\}$ provides only a small $\mathrm{O}(1)$ correction to the large wave number $\left(\ell_{p m} / \varepsilon\right) \breve{k}_{c}=\mathrm{O}\left(\delta^{-1 / 3} \varepsilon^{-1 / 3}\right)$ (see (84)). So essentially, each vortex lying under the pulse envelope propagates as a wave with phase velocity

$$
c_{n}=-\frac{\mathrm{x}_{n}(t)}{\left(\ell_{p m} / \varepsilon\right) \breve{k}_{c}} \quad \text { provided that } \quad\left|\mathrm{x}_{n}(t)\right|<\frac{\sqrt{\lambda}}{\ell_{S C}^{2} / \varepsilon}=\mathrm{O}\left(\delta^{2 / 3} \varepsilon^{-1 / 3}\right),
$$

where we have limited $x_{n}(t)$ in $(86 \mathrm{~b})$ by the requirement that the pulse lies within the unstable region. The estimate $(86 \mathrm{~b})$ implies that $c_{n}(86 \mathrm{a})$ is $\mathrm{O}(\delta)$, independent of $\varepsilon$, except of course near the equator $\mathrm{x}=0$. Furthermore, though the phase velocity $c_{n}$ and frequency $\mathrm{x}_{n}$ of each pulse (85) are discrete, at any location all pulses, linked to every frequency, exist. So it is not that the pulse centred on $\mathrm{x}=\mathrm{x}_{n}$ exists there in isolation, but rather that it dominates in the vicinity of $\mathrm{x}=\mathrm{x}_{n}$ with the magnitude of the other pulses (except possibly for its 
immediate neighbours) exponentially small. It is in that sense that the frequency staircase should be interpreted. Furthermore, though the contour plots of $\operatorname{Re}\{a(x, t)\}$ and $\operatorname{Im}\{a(x, t)\}$ in figure 5 describe $a(x, t)$ and illustrate nicely the frequency staircase effect, they provide little immediate quantitative information about the vortex amplitude $|a(x, t)|$, which undulates in amplitude and tends to peak locally near $x=x_{n}$. Such amplitude modulation is portrayed in figs. 21(d,f) of Harris et al. (2003) for the $\Upsilon=\frac{1}{4}, T=3.875$ case listed in our table 2 .

Staircase structures are surprisingly common in fluid mechanics. One such case with some common features concerns turbulence in rotating geophysical flows on a $\beta$-plane, where the potential vorticity $(\mathrm{PV})$ contains a dominant contribution from the vorticity of the rotating frame which varies linearly with latitude. The turbulence itself tends to generate shear flow (see, e.g., Rhines 1975, Vallis and Maltrud 1993) that modifies the PV to a staircase structure which is constant over a range of latitudes, but which jumps to new values across steps. With the PV constant over a distance, often referred to as the Rhines length scale (Rhines 1975), Rossby waves are suppressed except in the thin region where it jumps. The width of these steps is predictable up to an order of magnitude, but the reason for the particular realised values is unclear. We find a similar situation for our Taylor vortex frequency staircase, which again abhors continuous variation. The order of magnitude for the width of the frequency steps is predictable from balancing the phase mixing time $(T / \pi=) L^{-1}$ with the diffusion time $L^{2}$, which determines the latitudinal angular length scale $\ell_{p m}$ (see (23)) of the staircase but not the precise value realised, which may lie anywhere within a range which grows with increasing Taylor number. In short there is no predictable value and its realised value depends on the chaotic evolution.

Though much in this paper has been of a review nature, many of our results are contained in rather dense papers that are only penetrable by the most determined reader. The main purpose of our survey is to highlight the essential results for narrow-gap spherical Couette flow and to convey the main thrust of those developments to a wider audience who may find that the ideas and results relevant to them. To that end we have highlighted connections with the stability of other simple flows like plane Couette flow, which lack critical Reynolds numbers, as well as with staircase features common in geophysical flows. In so doing, we have provided novel and possibly significant interpretations and undertaken some extensions to previous published analysis.

Phase mixing is an ubiquitous phenomenon in both fluid dynamics and plasma physics with application to both geophysical and astrophysical flows (see, e.g., Heyvaerts and Priest 1983, Leprovost and Kim 2009, De Moortel and Nakariakov 2012, Rhines 1975, Farrell and Ioannou 1993, Vallis and Maltrud 1993, Soward 1977, Ewen and Soward 1994a,b, Dubrulle and Knobloch 1992, Hawley et al. 1995, Riols et al. 2013, Heinemann et al. 2011). Our CGLe (1) encapsulates the process in its purest form. In the absence of a critical value $\lambda$ for the bifurcation to non-trivial solutions, the simple pulse train solutions (75) are a paradigm for other more complex problems.

\section{Acknowledgement}

This paper was inspired by AMS' attendance (19-21 March 2014) of the LMS Society Conference "Nonlinear stability theory: from weakly nonlinear theory to the verge of turbulence" to celebrate the $85^{\text {th }}$ birthday of Professor J.T. Stuart. AMS gained further perspectives from attending (24-27 March 2014) the KITP program "Wave-flow interaction in geophysics, climate, astrophysics, and plasmas" at UCSB, where this research was supported in part by the National Science Foundation under Grant No. NSF PHY11-25915. We are grateful to the referees for their helpful comments. 


\section{References}

Abramowitz, M. and Stegun, I.A., NIST Handbook of Mathematical Functions. Eds. F.W.J. Olver, D.W. Lozier, R.F. Boisvert and C.W. Clark, CUP, NY 2010. (Available online http://dlmf.nist.gov/)

Bassom, A.P. and Soward, A.M., On finite amplitude subcritical instability in narrow gap spherical Couette flow. J. Fluid Mech. 2004, 499, 277-314.

Blockley, E., Bassom, A.P., Gilbert, A.D. and Soward, A.M., Pulse-train solutions of a spatially hetrogeneous amplitude equation arising in the subcritical instability of narrow gap spherical Couette flow. Physica $D$ 2007, 228, 1-30.

Busse, F.H., Bounds for turbulent shear flow. J. Fluid Mech. 1970, 41, 219-240.

Clever, R.M. and Busse, F.H., Three-dimensional convection in a horizontal layer subjected to constant shear. J. Fluid Mech. 1992, 234, 511-527.

Clever, R.M. and Busse, F.H., Tertiary and quaternary solutions for plane Couette flow. J. Fluid Mech. 1997, 344, 137-153.

Davey, A., The growth of Taylor vortices in flow between rotating cylinders. J. Fluid Mech. 1962, 14, 336-368.

De Moortel, I. and Nakariakov, V.M., Magnetohydrodynamic waves and coronal seismology: an overview of recent results. Phil. Trans. R. Soc. A 2012, 370, 3193-3216.

Dormy, E., Soward, A.M., Jones, C.A., Jault, D. and Cardin, P., The onset of thermal convection in rotating spherical shells. J. Fluid Mech. 2004, 501, 43-70.

Dubrulle, B. and Knobloch, E., On the local stability of accretion discs. Astron. Astrophys. 1992, 256, 673-678.

Eckhardt, B., Schneider, T.M., Hof, B. and Westerweel, J., Turbulence transition in pipe flow. Annu. Rev. Fluid Mech. 2007, 39, 447-468.

Ewen, S.A. and Soward, A.M., Phase mixed rotating magnetoconvection and Taylor's condition. II Travelling pulses. Geophys. Astrophys. Fluid Dyn. 1994a, 77, 231-262.

Ewen, S.A. and Soward, A.M., Phase mixed rotating magnetoconvection and Taylor's condition. III Wave trains. Geophys. Astrophys. Fluid Dyn. 1994b, 77, 263-283.

Faisst, H. and Eckhardt, B., Traveling waves in pipe flow. Phys. Rev. Lett. 2003, 91, 224502, 1-4.

Farrell, B.F. and Ioannou, P.J., Stochastic forcing of perturbation variance in unbounded shear and deformation flows. J. Atmos. Sci. 1993, 50, 200-211.

Harris, D., Bassom, A.P. and Soward, A.M., An inhomogeneous Landau equation with application to spherical Couette flow in the narrow gap limit. Physica D 2000, 137, 260-276.

Harris, D., Bassom, A.P. and Soward, A.M., Global bifurcation to travelling waves with application to narrow gap spherical Couette flow. Physica D 2003, 177, 122-174.

Hawley, J., Gammie, C.F. and Balbus, S.A., Local three-dimensional magnetohydrodynamic simulations of accretion disks. Astrophys. J. 1995, 440, 742-763.

Heinemann, T., McWilliams, J.C. and Schekochihin, A.A., Large-scale magnetic field generation by randomly forced shearing waves. Phys. Rev. Lett. 2011, 107, 255004, 1-5.

Heyvaerts, J. and Priest, E.R., Coronal heating by phase-mixed shear Alfvén waves. Astron. Astrophys. 1983, 117, 220-234.

Hocking, L.M., The instability of flow in the gap between two prolate spheroids. Part II. Arbitrary axis ratio. Q. Jl Mech. appl. Math. 1981, 34, 475-488.

Hocking, L.M. and Skiepko, J., The instability of flow in the gap between two prolate spheroids. Part I. Small axis ratio. Q. Jl Mech. appl. Math. 1981, 34, 57-68.

Joseph, D.D., Nonlinear stability of the Boussinesq equations by the method of energy. Arch. Rational Mech. Anal. 1966, 22, 163-184.

Koschmieder, E.L., Bénard Cells and Taylor Vortices. Cambridge University Press, Cambridge 1993

Leprovost, N. and Kim, E.J., Turbulent transport and dynamo in sheared magnetohydrodynamics turbulence with a nonuniform magnetic field. Phys. Rev. E 2009, 80, 255004, 1-12.

Manneville, P., On the transition to turbulence of wall-bounded flows in general, and plane Couette flow in particular. Eur. J. Mech. B-Fluid 2014, 49, 345-362.

Marcus, P.S. and Tuckerman, L.S., Simulation of flow between concentric rotating spheres. Part 2. Transitions. J. Fluid Mech. 1987, 185, 31-65.

Nagata, M., Three-dimensional finite-amplitude solutions in plane Couette flow: Bifurcation from infinity. J. Fluid Mech. 1990, 217, 519-527.

Rhines, P.B., Waves and turbulence on a beta-plane. J. Fluid Mech. 1975, 69, 417-443.

Riols, A., Rincon, F., Cossu, C., Lesur, G., Ogilvie, G.I., Longaretti, P.-Y. and Herault, J., Global bifurcations to subcritical magnetorotational dynamo action in Keplerian shear flow. J. Fluid Mech. 2013, 731, 1-45.

Soward, A.M., On the finite amplitude thermal instability of a rapidly rotating fluid sphere. Geophys. Astrophys. Fluid Dyn. 1977, 9, 19-74.

Soward, A.M. and Jones, C.A., The linear stability of the flow in the narrow gap between two concentric rotating spheres. Q. Jl Mech. appl. Math. 1983, 36, 19-42.

Squire, H.B., On the stability of three-dimensional disturbances of viscous flow between parallel walls. Proc. Roy. Soc. Lond. A 1933, 142, 621-628.

Stuart, J.T., On the non-linear mechanics of wave disturbances in stable and unstable parallel flows, Part 1. J. Fluid Mech. 1960, 9, 353-370.

Taylor, G.I., Stability of a viscous liquid contained between two rotating cylinders. 
Phil. Trans. Roy. Soc. Lond. A 1923, 223, 289-343.

Thomson, W. (Lord Kelvin), Stability of fluid motion - rectilinear motion of viscous fluid between two parallel planes. Phil. Mag. 1887, 24(5), 188-196.

Vallis, G.K. and Maltrud, M.E., Generation of mean flows and jets on a beta plane and over topography. J. Phys. Oceanogr. 1993, 23, 1346-1362.

Waleffe, F., On a self-sustaining process in shear flows. Phys. Fluids 1997, 9, 883-900.

Waleffe, F., Homotopy of exact coherent structures in plane shear flows. Phys. Fluids 2003, 15, 1517-1534.

Walton, I.C., The linear instability of flow in the narrow spherical annulus. J. Fluid Mech. 1978, 86, 673-693.

Wedin, H. and Kerswell, R.R., Exact coherent structures in pipe flow: travelling wave solutions. J. Fluid Mech. 2004, 508, 333-371.

\section{Appendix A: An application of Poisson's summation formula}

The results of this Appendix pivot upon the use of Poisson's summation formula, which we write in the generalised function form

$$
\sum_{m=-\infty}^{\infty} \delta(\zeta-m)=\sum_{m=-\infty}^{\infty} \exp (-2 \pi \mathrm{i} m \zeta)
$$

where $\delta(\zeta)$ is the Dirac delta function (see, e.g., Abramowitz and Stegun 2010, eq. http://dlmf.nist.gov/1.8.E14).

We define the Fourier Transform of $a(x, t)$, given by (66) with $L T=\pi$, as

$$
\mathcal{F}\{a\}(k, t)=\frac{1}{\sqrt{2} L} \int_{-\infty}^{\infty} a(x, t) \mathrm{e}^{-\mathrm{i} k x} \mathrm{~d} x=\sqrt{2} T \sum_{n=-\infty}^{\infty} \mu_{n} \delta(k-n T-t) \widehat{A}(t+n T) .
$$

By the simple expedient of noting that the non-zero contributions from the terms $\widehat{A}(t+n T)$ in the sum on the right-hand side only occur when $k=n T+t$, we rewrite the right-hand side of (A.2) in the alternative form

$$
\mathcal{F}\{a\}(k, t)=\sqrt{2} T \sum_{n=-\infty}^{\infty} \mu_{n} \delta((k-t)-n T) \widehat{A}(k) .
$$

Noting that $\mu_{4 m+\alpha}=\mu_{\alpha}$, where $\mu_{0}=\mu_{1}=1$ and $\mu_{2}=\mu_{3}=-1$ for $\alpha=0,1,2,3$ and $m=\cdots,-2,-1,0,1,2 \cdots$, we set $n=4 m+\alpha$ and reorganise (A.3) into the form

$$
\mathcal{F}\{a\}(k, t)=\frac{1}{2 \sqrt{2}} \sum_{\alpha=0}^{3} \mu_{\alpha}\left[\sum_{m=-\infty}^{\infty} \delta\left(\frac{L}{4 \pi}(k-t)-\frac{1}{4} \alpha-m\right)\right] \widehat{A}(k),
$$

which, on application of Poisson's summation formula (A.1) with $\zeta=\frac{1}{4} \pi^{-1} L(k-t)-\frac{1}{4} \alpha$ to each of the four infinite sums $(\alpha=0,1,2,3)$, becomes

$$
\mathcal{F}\{a\}(k, t)=\frac{1}{2 \sqrt{2}} \sum_{\alpha=0}^{3} \sum_{m=-\infty}^{\infty} \mu_{\alpha} \exp \left(\frac{1}{2} \mathrm{i} \pi m \alpha\right) \exp \left(-\frac{1}{2} \mathrm{i} m L(k-t)\right) \widehat{A}(k) .
$$

We reverse the order of summation and evaluate the sum

$$
\sum_{\alpha=0}^{3} \mu_{\alpha} \exp \left(\frac{1}{2} \mathrm{i} \pi m \alpha\right)=\left(1+\mathrm{e}^{\mathrm{i} m \pi / 2}\right)\left(1-\mathrm{e}^{\mathrm{i} m \pi}\right)= \begin{cases}2(1 \pm \mathrm{i}) & m=2 n \pm 1 \\ 0 & m=2 n\end{cases}
$$


with $n=\cdots,-2,-1,0,1,2 \cdots$. What remains is

$$
\mathcal{F}\{a\}(k, t)=\sum_{n=-\infty}^{\infty} \exp \left((-1)^{n} \mathrm{i} \pi / 4\right) \exp \left(-\mathrm{i}\left(n+\frac{1}{2}\right) L(k-t)\right) \widehat{A}(k),
$$

with Fourier inverse

$$
\begin{aligned}
a(x, t) & =\frac{1}{\sqrt{2} T} \int_{-\infty}^{\infty} \mathcal{F}\{a\}(k, t) \mathrm{e}^{\mathrm{i} k x} \mathrm{~d} k \\
& =\sum_{n=-\infty}^{\infty} \exp \left((-1)^{n} \mathrm{i} \pi / 4\right) \exp \left(\mathrm{i}\left(n+\frac{1}{2}\right) L t\right) \bar{a}\left(x-\left(n+\frac{1}{2}\right) L\right),
\end{aligned}
$$

where we have noted that $\mathcal{F}\{\bar{a}\}(k)=\widehat{A}(k)$ (see (73a)). 